\title{
THE
}

$11-15-1998$

\section{Transport Equation and Diffusion in Ultrathin Channels and Films}

\author{
A. E. Meyerovich \\ University of Rhode Island, sfo101@uri.edu
}

A. Stepaniants

University of Rhode Island

Follow this and additional works at: https://digitalcommons.uri.edu/phys_facpubs

Terms of Use

All rights reserved under copyright.

\section{Citation/Publisher Attribution}

Meyerovich, A. E., \&.Stepaniants, A.. (1998). Transport Equation and Diffusion in Ultrathin Channels and Films. Phys. Rev. B, 58(13), 13242-13263. doi: 10.1103/PhysRevB.58.13242

Available at: http://dx.doi.org/10.1103/PhysRevB.58.13242

This Article is brought to you for free and open access by the Physics at DigitalCommons@URI. It has been accepted for inclusion in Physics Faculty Publications by an authorized administrator of DigitalCommons@URI. For more information, please contact digitalcommons-group@uri.edu. 


\title{
Transport equation and diffusion in ultrathin channels and films
}

\author{
A. E. Meyerovich and A. Stepaniants \\ Department of Physics, University of Rhode Island, Kingston, Rhode Island 02881
}

(Received 6 May 1998)

\begin{abstract}
A rigorous perturbative transport equation for ballistic particles in thin films with random rough walls is derived by the diagrammatic Keldysh technique for both quasiclassical and quantized motion across the film. The derivation is based on canonical Migdal transformation that replaces a transport problem with random rough walls by an equivalent problem with flat boundaries and randomly distorted bulk. The rigorous derivation requires a modification of our previously used transformation to avoid non-Hermitian perturbations. The unusual nondiagonal structure of the effective scattering operator makes the transport equation different from the standard Waldmann-Snider equation when the distance between quantized levels for the motion across the film is comparable to the wall-induced perturbation. Outside of this anomalous quantum resonance region, the transport equation is similar to that for scattering by bulk impurities. The magnitude of the anomaly is calculated for degenerate particles and Gaussian correlations of surface inhomogeneities. The transport problem is solved analytically for the single-band occupancy and in the limiting cases of very large and very small correlation radii of inhomogeneities for an arbitrary correlation function of surface roughness. Elsewhere, the transport equation is analyzed numerically for the Gaussian correlation function. Transport coefficients are expressed explicitly via the angular harmonics of the surface correlation function; in the anomalous region, the results contain certain supplemental correlators. The results reveal various effects of interwall correlations on transport including an oscillatory dependence on the number of occupied minibands. The transition from quantum to quasiclassical description of ballistic motion across the (thick) film can be hindered by residual interwall interference effects similar to those in classical optic problems for thick films without bulk attenuation. Erroneous matrix elements in our previous calculations have been corrected. [S0163-1829(98)05943-8]
\end{abstract}

\section{INTRODUCTION}

Interaction of particles and waves with random rough walls has been studied for decades in various contexts from light and sound propagation to electron transport to gas dynamics and hydrodynamics (see Refs. 1-7). Repeated scattering of particles by random surface inhomogeneities leads to the formation of the mean free path, quantum interference effects, and localization. If the inhomogeneities are small, the quantum interference is weak and the wall-imposed localization length is large. ${ }^{8-13}$ Thus, the localization effects should be studied after or in the frame of the diffusion problem. ${ }^{14-16}$ The aim of this paper is to describe diffusion flows between rough walls in thin films, wires, layers, quantum wells, waveguides, etc., explicitly via the statistic and geometric properties of wall roughness, namely, via the correlation function of surface inhomogeneities.

Recent advances in molecular-beam epitaxy, quantum waveguides and wells, and nanotechnology have renewed interest in this transport problem (early data are reviewed in Refs. 17 and 18; for recent data, see Refs. 19-34 and references therein). Many theoretical approaches to flows along rough walls involve either oversimplified specular, diffuse, or Fuchs boundary conditions, or overcomplicated integrodifferential boundary conditions ${ }^{2-6,35,36}$ often ignoring the geometric and statistic properties of the surface. Perturbative expansions for weak roughness (see, e.g., Refs. 1, 2, 6, 7, 17, $27,28,33,34,37,38$, and references therein) can take the shape of the surface into account, though the main attention is usually paid to the scattering $S$ matrix rather than to the transport equation which is assumed to have a standard bulklike form. This assumption is often wrong. Below we derive and solve a rigorous quantum transport equation specifically for the situation when the scattering by random surface inhomogeneities is the main scattering mechanism. We introduce explicitly an effective bulk perturbation operator, which is exactly equivalent to the boundary problem with slight roughness, and derive a diagrammatic transport equation for this random operator. The transport equation is quantum in the direction perpendicular to the walls and is quasiclassical along the walls.

Ballistic transport between rough walls is determined by correlation of consecutive particle collisions with the opposite walls and by quantum multiple scattering by the same wall. These effects depend differently on intrawall and interwall correlations of surface inhomogeneities, and are determined by three spatial scales-the particle wavelength $\lambda$, distance between the walls $L$, and the correlation radii $R_{i j}$ of intrawall and interwall correlations. Our description of ballistic transport between random rough walls is valid for an arbitrary combination of these scales. The transport equation is solved, where possible analytically, in a wide range of parameters.

The main finding is that there is a range of parameters in which the quantum transport problem between rough walls is qualitatively different from transport with bulk impurity scattering and all previous versions of the transport equation $^{39,23,27,28,34,40}$ fail. The anomaly occurs when the distance between the quantized energy levels $\Omega_{j j^{\prime}}$ for the motion across the film is comparable to the collision operator, $\widehat{1 / \tau} \Omega \sim 1$. In this quantum resonance region, transport cannot be described with the help of any single-wall $S$ matrix and bulklike Boltzmann-Waldmann-Snider transport equation. In effect, our results provide the interference $S$ matrix for scat- 
tering from two opposite walls with the quantized motion between them. In a wide range of parameters, this $S$ matrix is simple despite the interference of scattering from the opposite walls. In the anomalous range, the $S$ matrix is complicated and involves new supplemental correlators. The operator structure of this $S$ matrix requires a major change in the form of the transport equation. Since flow channels are always restricted by two opposite boundaries, this anomaly is unavoidable in transport problems in the absence of bulk attenuation. We demonstrate how to solve this transport problem, and find the scope of deviation from the results based on a standard transport equation. Our procedure is mathematically rigorous, and all the restrictions are readily quantified. Unfortunately, all calculations for the anomalous region are extremely cumbersome.

In addition, we performed a systematic analysis of the transport manifestations of possible interwall correlations of surface inhomogeneities. These effects are more pronounced for quantized motion perpendicular to the walls. Sometimes, the interwall interference suppresses the quasiclassical limit for ballistic particles even for thick films.

Note that, in contrast, for example, to the diffraction problem or propagation through the layered media, the most important transport calculations for narrow channels are those for slight roughness. If the roughness is strong, the particles are dephased by each wall collision, and the transport problem becomes trivial. Formally, the results below are obtained for thin $2 \mathrm{D}$ films or quantum wells with random rough walls. These results, with minor modifications, can be applied to narrow 1D channels as long as we do not consider localization effects caused by scattering from random inhomogeneities, which are, of course, much stronger in 1D channels than in 2D films.

In the next section we discuss rigorous mapping of the boundary scattering problem onto the bulk. The standard diagrammatic technique operates with Hermitian operators and requires the Jacobian of mapping transformation to be $J$ $=1$. Therefore, we are forced to modify our original mapping transformation ${ }^{40-42}$ to ensure that the Jacobian $J=1$ and all operators are Hermitian. This modification becomes important in the anomalous region, and is unnecessary outside of it. In Sec. II we also correct an error in our previous expressions for matrix elements.

The diagrammatic transport equation for ballistic transport is derived in Sec. III with an emphasis on the deviation from the standard Boltzmann-Waldmann-Snider form when the gaps between the quantized levels for finite motion across the film are comparable to the wall-induced effective perturbation. The transport equation is compared with the one for the scattering by bulk impurities in quantum and quasiclassical limits.

In Sec. IV the transport equation is solved analytically in the limiting cases of large and small correlation radii of surface inhomogeneities. In Sec. V we calculate the transport coefficients, where possible analytically, for the Gaussian correlation of surface inhomogeneities in a wide range of parameters in quantum and quasiclassical regimes. In the anomalous region, we compare numerical results based on exact and standard transport equations. The analytical and numerical calculations reveal the nontrivial effect of interwall correlations on transport. The comparison with other recent transport data is discussed in a separate subsection. The final section presents the summary of our results.

\section{MAPPING OF BOUNDARY SCATTERING ONTO THE BULK}

One of the simplest rigorous derivations of the scattering operator for random rough walls is based on a coordinate transformation that flattens the boundary and transfers inhomogeneities to the bulk equations of motion. This idea has been used for electromagnetic and acoustic wave scattering, diffraction patterns, wave guides, etc., for many years (see Refs. 2, 6, 8, 9, 39, 43-49, and references therein). One of the earliest examples is the Migdal transformation in nuclear physics. The use of the Migdal-like transformation for transport problems was discussed in Refs. 50 and 51, but without an explicit expression for the coordinate transformation. Independently, ${ }^{40-42}$ we suggested an explicit form of a necessary coordinate transformation and expressed transport and localization parameters for ballistic transport in systems with random rough walls explicitly via the wall profile, namely, via the correlation function of wall inhomogeneities. Later, the same transformation was used for conductance in 1D rough cylindrical wires. ${ }^{52}$

This mapping of the random boundary problem onto a problem with a random bulk Hamiltonian provides a basis for a rigorous perturbation expansion for slight roughnessroughness with small amplitude and aperture of surface inhomogeneities. What is missing is a rigorous transport equation for such a Hamiltonian: our previous heuristic treatment in the frame of the same transport equation as for the usual bulk imperfections (impurities) is not always adequate because of peculiar operator properties of the effective collision operator. These anomalies are quite general and restrict application of other transport calculations. ${ }^{2,23,28,34,48}$

\section{A. Mapping transformation}

We consider an infinite 2D channel of the average thickness $L$ with rough boundaries

$$
x=L / 2-\xi_{1}(y, z), \quad x=-L / 2+\xi_{2}(y, z) .
$$

The inhomogeneities are small, $\xi_{1}(y, z), \xi_{2}(y, z) \ll L$, and random, $\left\langle\xi_{1}\right\rangle=\left\langle\xi_{2}\right\rangle=0$, with the correlation function

$$
\begin{aligned}
\zeta_{i k}(|\mathbf{s}|) & =\left\langle\xi_{i}\left(\mathbf{s}_{1}\right) \xi_{k}\left(\mathbf{s}_{1}+\mathbf{s}\right)\right\rangle \equiv \int \xi_{i}\left(\mathbf{s}_{1}\right) \xi_{k}\left(\mathbf{s}_{1}+\mathbf{s}\right) d \mathbf{s}_{1}, \\
\zeta_{i k}(\mathbf{q}) & =\int d^{2} s e^{i \mathbf{q} \cdot \mathbf{s} / \hbar} \zeta_{i k}(\mathbf{s})=\xi_{i}(\mathbf{q}) \xi_{k}(-\mathbf{q}),
\end{aligned}
$$

where $\mathbf{s}$ and $\mathbf{q}$ are the $2 \mathrm{D}$ vectors in the wall plane $y, z$. The correlation functions $\zeta_{11}$ and $\zeta_{22}$ describe intrawall correlations of inhomogeneities, and $\zeta_{12}=\zeta_{21}$ describe interwall correlations. The second equation in Eqs. (2) is valid for $\zeta_{12}$ only if opposite walls are correlated; otherwise, $\zeta_{12}=0$. Our classical results ${ }^{42}$ contained only the overall width of the channel $\xi_{+}(\mathbf{s})=\xi_{1}+\xi_{2}$ and the correlator $\zeta_{+}=\left\langle\xi_{+}\left(\mathbf{s}_{1}\right) \xi_{+}\left(\mathbf{s}_{2}\right)\right\rangle=\zeta_{11}+\zeta_{22}+2 \zeta_{12}$. In Ref. 40 , we assumed that this result holds for quantum transport as well. As we will see, for quantized motion one should retain both $\xi_{+}$ and $\xi_{-}=\xi_{2}-\xi_{1}$. 
We will express the transport coefficients via the angular harmonics of an arbitrary set of correlation functions $\zeta_{i k}(\mathbf{s})$, and supplement general expressions by computations for Gaussian correlations,

$$
\begin{aligned}
& \zeta_{i k}(\mathbf{s})=l_{i k}^{2} \exp \left(-s^{2} / 2 R_{i k}^{2}\right), \\
& \zeta_{i k}(\mathbf{q})=2 \pi l_{i k}^{2} R_{i k}^{2} \exp \left(-q^{2} R_{i k}^{2} / 2\right)
\end{aligned}
$$

including the case of small correlation radius $R \rightarrow 0$, i.e., the $\delta$-type correlations,

$$
\zeta_{i k}(\mathbf{s})=l_{i k}^{2} R_{i k}^{2} \delta(s) / s, \quad \zeta_{i k}(\mathbf{q})=2 \pi l_{i k}^{2} R_{i k}^{2} .
$$

To avoid the parameter clutter, in numerical calculations we assume that all three correlation lengths are the same, $R_{11}$ $=R_{22}=R_{12}=R$, while the amplitudes $a_{i k}, l_{i k}=\sqrt{a_{i k}} l$, with some typical scale $l$, may be different.

In Refs. 41, 42, and 40 we used the coordinate transformation

$$
X=\frac{L\left[x-\xi_{-}(y, z) / 2\right]}{L-\xi_{+}(y, z)}
$$

which made the walls (1) flat, $X= \pm L / 2$, and did not change the coordinates along the walls, $Y=y, Z=z$. The Jacobian $J$ of such transformation $J \neq 1$ and the Hamiltonian $\hat{H}=p^{2} / 2 m$ in momentum variables $\left\{P_{x, y, z}\right\}$ canonically conjugate to $\{X, Y, Z\}$ contained the non-Hermitian terms. We dealt with this either by symmetrizing the perturbation $\hat{V} \rightarrow\left(\hat{V}+\hat{V}^{\dagger}\right) / 2$ in Refs. 41 and 42 , or by ignoring the nonHermitian nature of $\hat{V}$ in Ref. 40. As we will see, both approaches are correct in the range of parameters in which the transport equation keeps its standard form. Beyond this range, the symmetry properties of the perturbation are crucial.

Rigorous diagrammatic derivation requires that all operators are Hermitian and coordinate transformations have the Jacobian $J=1$. Thus, we are forced to supplement the stretching of the film thickness (5) by isotropic stretching of coordinates $(y, z)$ along the film to have $J=1$ :

$$
\begin{gathered}
Y=y[1+\gamma(\mathbf{s})], \quad Z=z[1+\gamma(\mathbf{s})], \\
\gamma(\mathbf{s})=-\frac{1}{L} \int_{0}^{1} \xi_{+}(\alpha \mathbf{s}) \alpha d \alpha .
\end{gathered}
$$

\section{B. Effective Hamiltonian and scattering probabilities}

In new momentum variables $\mathbf{p} \rightarrow \mathbf{P}$, which are conjugate to Eqs. (5) and (6), the bulk Hamiltonian $\hat{H}=p^{2} / 2 m$ acquires the form

$$
\begin{aligned}
& \hat{H}=\frac{\hat{P}^{2}}{2 m}+\hat{V}, \quad \hat{V}=\hat{V}_{x}+\hat{V}_{y}+\hat{V}_{z}+\hat{V}_{2}, \quad \hat{V}_{x}=\frac{\xi_{+}}{m L} \hat{P}_{x}^{2}, \\
& \hat{V}_{i}=\frac{1}{2 m}\left[\left(\frac{X}{L} \xi_{+i}^{\prime}-\frac{1}{2} \xi_{-i}^{\prime}\right) \hat{P}_{x} \hat{P}_{i}+\hat{P}_{i}\left(\frac{X}{L} \xi_{+i}^{\prime}-\frac{1}{2} \xi_{-i}^{\prime}\right) \hat{P}_{x}\right],
\end{aligned}
$$

$$
\begin{aligned}
\hat{V}_{2}= & \frac{1}{m}\left[\gamma(\mathbf{s}) \hat{P}_{i} \hat{P}_{i}+i s_{k}\left(\hat{P}_{i} \gamma(\mathbf{s})\right) \hat{P}_{i} \hat{P}_{k}+\left(\hat{P}_{i} \gamma(\mathbf{s})\right) \hat{P}_{i}\right. \\
& \left.+\frac{1}{2} i s_{k}\left(\hat{P}_{i} \hat{P}_{i} \gamma(\mathbf{s})\right) \hat{P}_{k}\right],
\end{aligned}
$$

where the indices $(i, k)$ take the values $(y, z)$. Equation (7) differs from Refs. 40-42 by the presence of $\xi_{-}$and the integral terms with $\gamma(\mathbf{s})$ originating from Eq. (6). The perturbation expansion with the Hamiltonian (7) linearized in $\xi$ requires small and smooth inhomogeneities,

$$
l \ll L, R \text {. }
$$

The condition $l \ll R$ does not restrict our approach to the large-size inhomogeneities. The physical scale for the correlation range is defined by the particles wavelength $\lambda$ and not by the height of inhomogeneities $l$. For longwave particles $\lambda \gg R$, the correlations are essentially $\delta$-like (4). The exact profile of $\zeta_{i k}$ is important only for $\lambda<R$.

The non-Hermitian operator $\hat{X} \hat{P}_{x}$ in $\hat{V}_{i}(7)$ is the source of all anomalies. The terms with $\gamma$ in Eq. (7), related to the transformation (6), make the full perturbation $\hat{V}$ Hermitian at a price of more cumbersome equations than Refs. 40-42. The nondiagonal structure of the scattering vertex, which is determined by $\hat{V}$, hinders the derivation of the transport equation. This complication is not an accidental peculiarity of our method. The off-diagonal terms reflect the fact that the film thickness is changing randomly along the film leading to the violation of the conservation law for $P_{x}$ even after averaging. As a result, the scattering vertex is always nondiagonal with nontrivial operator properties. ${ }^{50,53}$

The transformations (5) and (6) reduce the transport problem between rough walls to an equivalent transport problem with ideal specular walls $\Psi(L / 2)=\Psi(-L / 2)=0$ and distorted bulk Hamiltonian (7). In thin films, the unperturbed motion of particles across the films is quantized, $\left(P_{x}\right)_{j}$ $=\pi j / L$, with the wave functions

$$
\Psi_{j}(\mathbf{r})=\sqrt{2 / v_{0}} \exp (i \mathbf{q} \cdot \mathbf{s}) \sin [\pi j(X+L / 2) / L]
$$

( $v_{0}$ is the volume). The matrix elements of $\hat{V}$ are

$$
\begin{aligned}
V_{\mathbf{q} j, \mathbf{q}^{\prime} j^{\prime}}= & \frac{\delta_{j j^{\prime}}}{m L}\left[\xi_{+}\left(\mathbf{q}^{\prime}-\mathbf{q}\right)\left(\frac{\pi j}{L}\right)^{2}+\frac{1}{4}\left(q^{\prime 2}-q^{2}\right)\left(\mathbf{q}^{\prime}+\mathbf{q}\right) \cdot \frac{\partial}{\partial\left(\mathbf{q}^{\prime}-\mathbf{q}\right)} \gamma\left(\mathbf{q}^{\prime}-\mathbf{q}\right)\right] \\
& -\frac{1-\delta_{j j^{\prime}}}{m L} \frac{j j^{\prime}}{j^{\prime 2}-j^{2}}\left(q^{\prime 2}-q^{2}\right)\left[\xi_{+}\left(\mathbf{q}^{\prime}-\mathbf{q}\right) \frac{1+(-1)^{j+j^{\prime}}}{2}+\xi_{-}\left(\mathbf{q}^{\prime}-\mathbf{q}\right) \frac{1-(-1)^{j+j^{\prime}}}{2}\right],
\end{aligned}
$$




$$
\gamma(\mathbf{q})=-\frac{1}{L} \int_{1}^{\infty} \xi_{+}(\alpha \mathbf{q}) \frac{d \alpha}{\alpha}
$$

Even without the terms with $\gamma$ and $\xi_{-}$, the matrix elements (10) are slightly different from those in Refs. 42 and 40 because of an error in our previous expressions. The randomness of inhomogeneities, $\langle\xi\rangle=0$, means that $\langle\hat{V}\rangle_{\xi}=0$. All nonvanishing averages start from the quadratic combinations of the matrix elements $V_{\mathbf{q} j, \mathbf{q}^{\prime} j^{\prime}}$.

The transition probabilities $W$ in the collision operator in the transport equation are quadratic in $\hat{V}$ (10) and can be expressed, after averaging over random functions $\xi$, via the correlation functions $\zeta$ :

$$
\begin{aligned}
& \left\langle V_{\mathbf{q}_{1} j_{1}, \mathbf{q}_{1}^{\prime} j_{1}^{\prime}} V_{\mathbf{q}_{1}^{\prime} j_{2}, \mathbf{q}_{2}^{\prime} j_{2}^{\prime}}\right\rangle_{\xi}=(2 \pi)^{2} W_{j_{1} j_{1}^{\prime} j_{2} j_{2}^{\prime}}^{(1)}\left(\mathbf{q}_{1}, \mathbf{q}_{1}^{\prime}\right) \delta\left(\mathbf{q}_{1}-\mathbf{q}_{2}^{\prime}\right),
\end{aligned}
$$

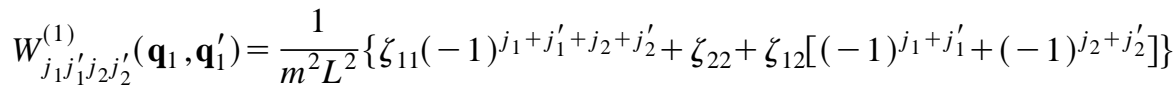

$$
\begin{aligned}
& \times\left\{\left[\left(\frac{\pi j_{1}}{L}\right)^{2}-\frac{1}{4} \frac{\left(q_{1}^{2}-q_{1}^{\prime 2}\right)^{2}}{\left(\mathbf{q}_{1}^{\prime}-\mathbf{q}_{1}\right)^{2}}\right] \delta_{j_{1} j_{1}^{\prime}}+\left(q_{1}^{2}-q_{1}^{\prime 2}\right) \frac{\left(1-\delta_{j_{1} j_{1}^{\prime}}\right) j_{1} j_{1}^{\prime}}{j_{1}^{\prime 2}-j_{1}^{2}}\right\} \\
& \times\left\{\left[\left(\frac{\pi j_{2}}{L}\right)^{2}-\frac{1}{4} \frac{\left(q_{1}^{2}-q_{1}^{\prime 2}\right)^{2}}{\left(\mathbf{q}_{1}^{\prime}-\mathbf{q}_{1}\right)^{2}}\right] \delta_{j_{2} j_{2}^{\prime}}-\left(q_{1}^{2}-q_{1}^{\prime 2}\right) \frac{\left(1-\delta_{j_{2} j_{2}^{\prime}}\right) j_{2} j_{2}^{\prime}}{j_{2}^{\prime 2}-j_{2}^{2}}\right\} \\
& +\frac{\delta_{j_{1} j_{1}^{\prime}} \delta_{j_{2} j_{2}^{\prime}}}{4 m^{2} L^{2}}\left[\frac{\widetilde{\zeta}_{11}^{2}}{\zeta_{11}}+\frac{\widetilde{\zeta}_{22}^{2}}{\zeta_{22}}+2 \frac{\widetilde{\zeta}_{11} \widetilde{\zeta}_{22}}{\sqrt{\zeta_{11} \zeta_{22}}}\right] \frac{\left(q_{1}^{2}-q_{1}^{\prime 2}\right)^{2}}{\left(\mathbf{q}_{1}^{\prime}-\mathbf{q}_{1}\right)^{4}}\left[q_{1}^{2} q_{1}^{\prime 2}-\left(\mathbf{q}_{1}^{\prime} \cdot \mathbf{q}_{1}\right)^{2}\right],
\end{aligned}
$$

where \langle\rangle$_{\xi}$ stands for the averaging over $\xi$, the "supplemental" correlation functions $\widetilde{\zeta}_{i i}$ are

$$
\begin{aligned}
& \widetilde{\zeta}_{i i}(\mathbf{q})=\widetilde{\zeta}_{i i}(-\mathbf{q})=\xi_{i}(\mathbf{q}) \widetilde{\xi}_{i}(-\mathbf{q}), \\
& \widetilde{\xi}_{i}(\mathbf{q})=q \mathbf{e} \cdot \frac{\partial}{\partial \mathbf{q}} \int_{1}^{\infty} \xi_{i}(\alpha \mathbf{q}) \frac{d \alpha}{\alpha},
\end{aligned}
$$

$\mathbf{e}$ is the unit vector perpendicular to $\mathbf{q}$ in the plane of the wall (since $W$ is quadratic in e, a choice of two possible orientations of $\mathbf{e}$ is irrelevant), and the correlation functions in Eq. (11) are $\zeta_{i k}\left(\mathbf{q}_{1}^{\prime}-\mathbf{q}_{1}\right)$ and $\widetilde{\zeta}_{i k}\left(\mathbf{q}_{1}^{\prime}-\mathbf{q}_{1}\right)$. $W_{j_{1} j_{1}^{\prime} j_{2} j_{2}^{\prime}}^{(1)}(11)$ is only a part of the transition probability $W_{j_{1} j_{1}^{\prime} j_{2} j_{2}^{\prime}}=W_{j_{1} j_{1}^{\prime} j_{2} j_{2}^{\prime}}^{(1)}+W_{j_{1} j_{1}^{\prime} j_{2} j_{2}^{\prime}}^{(2)}$; the expression for $W_{j_{1} j_{1}^{\prime} j_{2} j_{2}^{\prime}}^{(2)}$ is given in the Appendix.

\section{Standard Waldmann-Snider transport equation}

Heuristically, ${ }^{40-42}$ one can treat $\hat{V}(7)$ within the Boltzmann equation for particles with minibands $\epsilon_{j \mathbf{q}}=\left[(\pi j / L)^{2}\right.$ $\left.+q^{2}\right] / 2 m$ with discrete quantum number $j$

$$
\partial_{t} \delta n\left(\epsilon_{j}, \mathbf{q}\right)+\frac{\mathbf{q}}{m} \cdot \partial_{\mathbf{r}} \delta n\left(\epsilon_{j}, \mathbf{q}\right)+\mathbf{F} \cdot \partial_{\mathbf{q}} \delta n\left(\epsilon_{j}, \mathbf{q}\right)=L_{j}\left\{n_{i}\right\}
$$

Equation (13) is often called the Waldmann-Snider equation. ${ }^{54}$ The perturbative collision integrals $L_{j}$ are determined by the transition probabilities $W_{j j^{\prime}}\left(\mathbf{q}, \mathbf{q}^{\prime}\right) \equiv W_{j j^{\prime} j^{\prime} j}\left(\mathbf{q}, \mathbf{q}^{\prime}\right)=\left\langle\left|V_{j \mathbf{q}, j^{\prime} \mathbf{q}^{\prime}}\right|^{2}\right\rangle$ between the states $(j, \mathbf{q}) \rightarrow\left(j^{\prime}, \mathbf{q}^{\prime}\right)$,

$$
L_{j}=2 \pi \sum_{j^{\prime}} \int W_{j j^{\prime}}\left(\mathbf{q}, \mathbf{q}^{\prime}\right)\left[n_{j^{\prime}}^{\prime}-n_{j}\right] \delta\left(\boldsymbol{\epsilon}_{j \mathbf{q}}-\boldsymbol{\epsilon}_{j^{\prime} \mathbf{q}^{\prime}}\right) \frac{d^{2} q^{\prime}}{(2 \pi)^{2}} .
$$

Equation (11) gives the part $W_{j j^{\prime} j^{\prime} j}^{(1)}\left(\mathbf{q}, \mathbf{q}^{\prime}\right)$ of the full transition probability $W=W^{(1)}+W^{(2)}$ in Eq. (14):

$$
\begin{aligned}
W_{j j^{\prime} j^{\prime} j}^{(1)}\left(\mathbf{q}, \mathbf{q}^{\prime}\right)= & \frac{1}{m^{2} L^{2}}\left[\zeta_{11}+\zeta_{22}+2 \zeta_{12}(-1)^{j+j^{\prime}}\right] \\
& \times\left\{\left[\left(\frac{\pi j}{L}\right)^{2}-\frac{1}{4} \frac{\left(q^{2}-q^{\prime 2}\right)^{2}}{\left(\mathbf{q}^{\prime}-\mathbf{q}\right)^{2}}\right]^{2} \delta_{j j^{\prime}}+\left(q^{2}-q^{\prime 2}\right)^{2} \frac{\left(1-\delta_{j j^{\prime}}\right) j^{2} j^{\prime 2}}{\left(j^{\prime 2}-j^{2}\right)^{2}}\right\} \\
& +\frac{\delta_{j j^{\prime}}}{4 m^{2} L^{2}}\left[\frac{\widetilde{\zeta}_{11}^{2}}{\zeta_{11}}+\frac{\widetilde{\zeta}_{22}^{2}}{\zeta_{22}}+2 \frac{\widetilde{\zeta}_{11} \widetilde{\zeta}_{22}}{\sqrt{\zeta_{11} \zeta_{22}}}\right] \frac{\left(q^{2}-q^{\prime 2}\right)^{2}}{\left(\mathbf{q}^{\prime}-\mathbf{q}\right)^{4}}\left[q^{2} q^{\prime 2}-\left(\mathbf{q}^{\prime} \cdot \mathbf{q}\right)^{2}\right] .
\end{aligned}
$$


$W_{j j^{\prime} j^{\prime} j}^{(2)}$ can be found in the Appendix. Since the terms with $\left(q^{2}-q^{\prime 2}\right) \delta_{j j^{\prime}}$ do not contribute to Eq. (13) because of the energy $\delta$ functions $\delta\left(\epsilon_{j \mathbf{q}}-\epsilon_{j^{\prime} \mathbf{q}^{\prime}}\right)$ in the integrand (14), Eq. (15) can be simplified:

$$
W_{j j^{\prime} j^{\prime} j}^{(1)}\left(\mathbf{q}, \mathbf{q}^{\prime}\right)=\frac{1}{m^{2} L^{2}}\left[\zeta_{11}+\zeta_{22}+2 \zeta_{12}(-1)^{j+j^{\prime}}\right]\left(\frac{\pi j}{L}\right)^{2}\left(\frac{\pi j^{\prime}}{L}\right)^{2} .
$$

For the same reason, the terms with $W_{j j^{\prime} j^{\prime} j}^{(2)}\left(\mathbf{q}, \mathbf{q}^{\prime}\right)$ from the Appendix disappear completely from the Boltzmann-WaldmannSnider transport equation (13):

$$
L_{j}=\frac{1}{2 \pi m^{2} L^{2}} \sum_{j^{\prime}}\left(\frac{\pi j}{L}\right)^{2}\left(\frac{\pi j^{\prime}}{L}\right)^{2} \int d^{2} q^{\prime}\left[\zeta_{11}+\zeta_{22}+2 \zeta_{12}(-1)^{j+j^{\prime}}\right]\left[n_{j^{\prime}}\left(\mathbf{q}^{\prime}\right)-n_{j}(\mathbf{q})\right] \delta\left(\boldsymbol{\epsilon}_{j^{\prime} \mathbf{q}^{\prime}}-\boldsymbol{\epsilon}_{j \mathbf{q}}\right)
$$

(the difference from Refs. 41, 42, and 50 is explained by the above-mentioned difference in the matrix elements). The equation is quasiclassical along the walls and quantum in the transverse direction and requires, apart from condition (8), one of the two quasiclassical conditions

$$
l / L \ll q^{2} R^{2}, \quad q^{3} R L^{2} \text { or } 1 \ll q R .
$$

Note that the simplicity of the collision operator (17) is not accidental and is common to a wide range of problems with rough surfaces and interfaces as long as the collision operators contain the energy $\delta$ function. A similar transport equation was used recently, though without the interwall term, in Refs. 23, 28, and 34. The heuristic transport equations (13) and (17) can be wrong even under conditions (8) and (18).

\section{DERIVATION OF THE TRANSPORT EQUATION}

\section{A. Diagrammatic derivation}

To derive a rigorous transport equation for particles with the Hamiltonian (7) in finite films, we use the Keldysh procedure and notations of Ref. 55 in combination with the averaging technique. ${ }^{56}$ In principle, we could use alternative diagrammatic techniques. ${ }^{57,56}$ In multiband systems $\epsilon_{j \mathbf{q}}$, these techniques require an inversion of large matrices. In contrast to scattering by bulk impurities, these nondiagonal matrices cannot be inverted analytically, thus requiring numerical calculations from the start. The use of the transport equation postpones the inversion problem to the last stage, namely to the stage of solving the transport equation. This allows us to find an analytical dependence of transport coefficients on physical parameters.

In films with quantized motion $\left(P_{x}\right)_{j}$, the single-particle distribution is a matrix in the indices $j$,

$$
\begin{aligned}
n_{j j^{\prime}}(t, \mathbf{s}, \mathbf{q})= & -i \frac{2}{L} \lim _{t=t_{1}=t_{2}-0} \int G^{-+}\left(t_{1}, \mathbf{r}_{1} ; t_{2}, \mathbf{r}_{2}\right) \exp \left[-i \mathbf{q}\left(\mathbf{s}_{1}-\mathbf{s}_{2}\right)\right] \\
& \times \sin \left[\pi j\left(X_{1}-L / 2\right) / L\right] \sin \left[\pi j^{\prime}\left(X_{2}-L / 2\right) / L\right] d\left(\mathbf{s}_{1}-\mathbf{s}_{2}\right) d X_{1} d X_{2} .
\end{aligned}
$$

The Fourier image of the equilibrium Green's function $\hat{G}_{j j^{\prime}}^{(0)}$ without interaction $\hat{V}$ (7), is

$$
\hat{G}_{j j^{\prime}}^{(0)}\left(\omega_{1}, \mathbf{q}_{1} ; \omega_{2}, \mathbf{q}_{2}\right)=(2 \pi)^{3} \delta\left(\omega_{1}-\omega_{2}\right) \delta\left(\mathbf{q}_{1}-\mathbf{q}_{2}\right) \delta_{j j^{\prime}} \hat{G}_{j}^{(0)}\left(\omega_{1}, \mathbf{q}_{1}\right),
$$

where the \pm components of the matrix $\hat{G}_{j}^{(0)}$ are

$$
\begin{gathered}
G_{j}^{(0) \pm \pm}(\omega, \mathbf{q})=\frac{\mp 1}{\omega-\epsilon_{j \mathbf{q}}+\mu+i 0}-2 \pi i\left(\frac{1 \pm 1}{2}-n_{j}(\mathbf{q})\right) \delta\left(\omega-\boldsymbol{\epsilon}_{j \mathbf{q}}+\mu\right), \\
G_{j}^{(0) \pm \mp}(\omega, \mathbf{q})=2 \pi i\left(n_{j}(\mathbf{q})-\frac{1 \pm 1}{2}\right) \delta\left(\omega-\epsilon_{j \mathbf{q}}+\mu\right) .
\end{gathered}
$$

As usual, the transport equation is determined by the diagram in Fig. 1(a),

$$
\begin{aligned}
G_{j j^{\prime}}^{-+}\left(\omega, \mathbf{q} ; \omega^{\prime}, \mathbf{q}^{\prime}\right)= & G_{j j^{\prime}}^{(V)-+}\left(\omega, \mathbf{q} ; \omega^{\prime}, \mathbf{q}^{\prime}\right)+(2 \pi)^{2} \sum_{j_{1}, j_{1}^{\prime}, j_{2}, j_{2}^{\prime}} \int d \Gamma_{1} d \Gamma_{1}^{\prime} d \Gamma_{2} d \Gamma_{2}^{\prime} \delta\left(\omega_{1}-\omega_{1}^{\prime}\right) \delta\left(\omega_{2}-\omega_{2}^{\prime}\right) V_{\mathbf{q}_{1} j_{1}, \mathbf{q}_{1}^{\prime} j_{1}^{\prime}} V_{\mathbf{q}_{2} j_{2}, \mathbf{q}_{2}^{\prime} j_{2}^{\prime}} \\
& \times\left[\hat{G}_{j j_{1}}^{(V)}\left(\omega, \mathbf{q} ; \omega_{1}, \mathbf{q}_{1}\right) \hat{G}_{j_{1}^{\prime} j_{2}}^{(V)}\left(\omega_{1}^{\prime}, \mathbf{q}_{1}^{\prime} ; \omega_{2}, \mathbf{q}_{2}\right) \hat{G}_{j_{2}^{\prime} j^{\prime}}\left(\omega_{2}^{\prime}, \mathbf{q}_{2}^{\prime} ; \omega^{\prime}, \mathbf{q}^{\prime}\right)\right]^{-+}
\end{aligned}
$$

where the index $(V)$ in $\hat{G}_{j j_{1}}^{(V)}$ denotes the nonequilibrium Green's function without interaction $\hat{V}$ and $d \Gamma_{i}=d \omega_{i} d^{2} q_{i} /(2 \pi)^{3}$. In Fig. 1 , the indices $\alpha, \beta$ take the values \pm , while the numerical indices $1,1^{\prime}$, etc., correspond to $(j, \omega, \mathbf{q}),\left(j^{\prime}, \omega^{\prime}, \mathbf{q}^{\prime}\right)$, etc. The diagrams with an odd number of dashed (scattering) lines disappear after averaging over inhomogeneities and are not included in Fig. 1. 

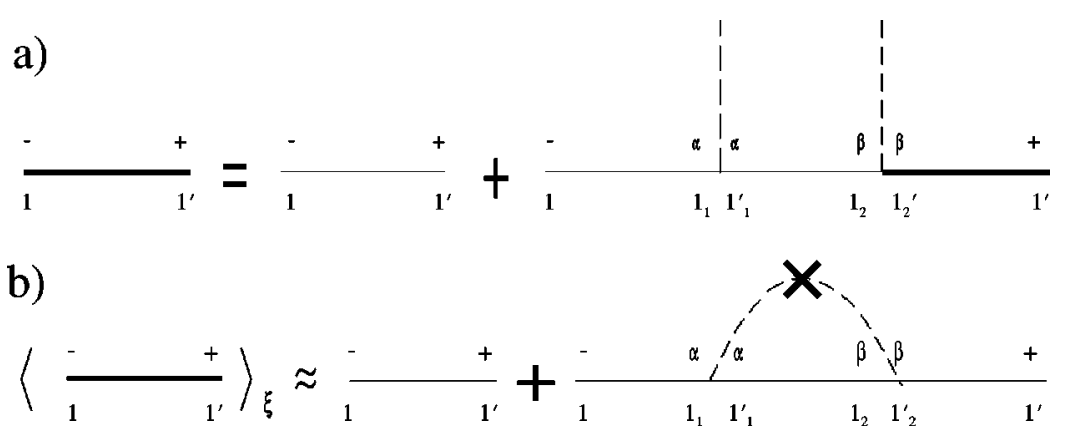

FIG. 1. Nonvanishing diagrams (a) before and (b) after averaging over surface inhomogeneities. The diagram (a) is exact; (b) is truncated in the second order.

The second relevant diagram differs from Fig. 1(a) by having the bold line to the left of the interaction. To get the transport equation, we multiply these two diagrams by $G^{(0)-1}$ (the first one by $\omega-\epsilon_{j \mathbf{q}}+\mu$, the second one by $\omega^{\prime}-\epsilon_{j^{\prime} \mathbf{q}^{\prime}}+\mu$ ), and subtract one from another. Then the left-hand side (LHS) becomes

$$
\left(\omega-\omega^{\prime}-\epsilon_{j \mathbf{q}}+\epsilon_{j^{\prime} \mathbf{q}^{\prime}}\right) G_{j j^{\prime}}^{-+}\left(\omega, \mathbf{q} ; \omega^{\prime}, \mathbf{q}^{\prime}\right) .
$$

After we separate slow and fast variables and perform the Fourier transform over the slow ones,

$$
G_{j j^{\prime}}^{-+}\left(\omega, \mathbf{q} ; \omega^{\prime}, \mathbf{q}^{\prime}\right)=G_{j j^{\prime}}^{-+}\left(\omega-\omega^{\prime}, \mathbf{q}-\mathbf{q}^{\prime} ; \frac{\omega+\omega^{\prime}}{2}, \frac{\mathbf{q}+\mathbf{q}^{\prime}}{2}\right)=\int \exp \left[i\left(\omega-\omega^{\prime}\right) t-i\left(\mathbf{q}-\mathbf{q}^{\prime}\right) \mathbf{s}\right] G_{j j^{\prime}}^{-+}\left(t, \mathbf{s} ; \frac{\omega+\omega^{\prime}}{2}, \frac{\mathbf{q}+\mathbf{q}^{\prime}}{2}\right) d t d \mathbf{s},
$$

Eq. (22) assumes the form

$$
\left(\partial_{t}+\frac{\mathbf{q}+\mathbf{q}^{\prime}}{2 m} \cdot \partial_{\mathbf{s}}+i \Omega_{j j^{\prime}}\right) G_{j j^{\prime}}^{-+}\left(t, \mathbf{s} ; \frac{\omega+\omega^{\prime}}{2}, \frac{\mathbf{q}+\mathbf{q}^{\prime}}{2}\right),
$$

where we multiplied the equation by $-i$ and introduced the interband transition frequencies

$$
\Omega_{j j^{\prime}}=\frac{1}{2 m}\left(\frac{\pi}{L}\right)^{2}\left[j^{2}-j^{\prime 2}\right]
$$

To get the equation for the distribution function from the equation for $G_{j j^{\prime}}^{-+}$, we integrate the equation over the fast frequency variable:

$$
n_{j j^{\prime}}\left(t, \mathbf{s} ; \frac{\mathbf{q}+\mathbf{q}^{\prime}}{2}\right)=-i \int \frac{d\left(\omega+\omega^{\prime}\right)}{4 \pi} G_{j j^{\prime}}^{-+}\left(t, \mathbf{s} ; \frac{\omega+\omega^{\prime}}{2}, \frac{\mathbf{q}+\mathbf{q}^{\prime}}{2}\right) .
$$

Finally, the LHS (24) of the transport equation reduces to a usual form

$$
\left(\partial_{t}+\frac{\mathbf{q}+\mathbf{q}^{\prime}}{2 m} \cdot \partial_{\mathbf{s}}+i \Omega_{j j^{\prime}}\right) n_{j j^{\prime}}\left(t, \mathbf{s} ; \frac{\mathbf{q}+\mathbf{q}^{\prime}}{2}\right) .
$$

The same operations should be performed over the collision operator on the right-hand side (RHS) of the equation.

\section{B. Collision operator}

In the lowest order in $\hat{V}$, we can disregard $\hat{V}$ in the remaining full Green's function $\hat{G}_{j_{2}^{\prime} j^{\prime}}\left(\omega_{2}^{\prime}, \mathbf{q}_{2}^{\prime} ; \omega^{\prime}, \mathbf{q}^{\prime}\right)$ in the RHS of Eq. (21), and replace $\hat{G}_{j_{2}^{\prime} j^{\prime}}$ by $\hat{G}_{j_{2}^{\prime} j^{\prime}}^{(V)}$. After this, the averaging over surface inhomogeneities \langle\rangle$_{\xi}$ [connecting the interaction lines in the diagram of Fig. 1(b)] becomes trivial since the only term that has to be averaged is the quadratic combination of the matrix elements of $\hat{V}(10)$.

Close to equilibrium, the kinetic equation can be linearized, $\hat{G}^{(V)}=\hat{G}^{(0)}+\delta \hat{G}$. Using the definition of the Green's functions $\hat{G}_{j j^{\prime}}^{(V)}$ via the construction operators $\hat{a}^{\dagger}, \hat{a}$, it is easy to show that, since $\hat{G}^{(V)}$ does not depend on interaction, all four $( \pm)$ components of $\delta \hat{G}$ are the same, $\delta G_{j j^{\prime}}^{ \pm \pm}=\delta G_{j j^{\prime}}^{\mp \pm}=\delta G_{j j^{\prime}}$. Then the RHS of the linearized transport equation (21) acquires the form

$$
\sum_{j_{1}^{\prime} j_{2}} \int \frac{d^{2} q_{1}^{\prime}}{(2 \pi)^{2}}\left[\begin{array}{c}
W_{j j_{1}^{\prime} j_{2} j^{\prime}}\left(\mathbf{q}, \mathbf{q}_{1}^{\prime}, \mathbf{q}^{\prime}+\mathbf{q}_{1}^{\prime}-\mathbf{q}, \mathbf{q}^{\prime}\right) \delta G_{j_{1}^{\prime} j_{2}}\left(\omega, \mathbf{q}_{1}^{\prime} ; \omega^{\prime}, \mathbf{q}^{\prime}+\mathbf{q}_{1}^{\prime}-\mathbf{q}\right)\left[G_{j^{\prime}}^{A}\left(\omega^{\prime}, \mathbf{q}^{\prime}\right)-G_{j}^{R}(\omega, \mathbf{q})\right] \\
+W_{j j_{1}^{\prime} j_{1}^{\prime} j_{2}}\left(\mathbf{q}, \mathbf{q}_{1}^{\prime}, \mathbf{q}_{1}^{\prime}, \mathbf{q}\right) \delta G_{j_{2} j^{\prime}}\left(\omega, \mathbf{q} ; \omega^{\prime}, \mathbf{q}^{\prime}\right) G_{j_{1}^{\prime}}^{R}\left(\omega, \mathbf{q}_{1}^{\prime}\right) \\
-W_{j_{2} j_{1}^{\prime} j_{1}^{\prime} j^{\prime}}\left(\mathbf{q}^{\prime}, \mathbf{q}_{1}^{\prime}, \mathbf{q}_{1}^{\prime}, \mathbf{q}^{\prime}\right) \delta G_{j j_{2}}\left(\omega, \mathbf{q} ; \omega^{\prime}, \mathbf{q}^{\prime}\right) G_{j_{1}^{\prime}}^{A}\left(\omega^{\prime}, \mathbf{q}_{1}^{\prime}\right)
\end{array}\right],
$$

where $G_{j}^{R}(\omega, \mathbf{q})$ and $G_{j}^{A}(\omega, \mathbf{q})$ are the usual unperturbed retarded and advanced Green's functions, 


$$
G_{j}^{A, R}(\omega, \mathbf{q})=\frac{1}{\omega-\epsilon_{j \mathbf{q}}+\mu \bar{\mp} i 0} \equiv P \frac{1}{\omega-\epsilon_{j \mathbf{q}}+\mu} \pm i \pi \delta\left(\omega-\epsilon_{j \mathbf{q}}+\mu\right) .
$$

After we separate fast and slow variables as in Eq. (23),

$$
\delta G_{j j^{\prime}}\left(\omega_{1}, \mathbf{q}_{1} ; \omega_{2}, \mathbf{q}_{2}\right)=(2 \pi)^{3} g_{j j^{\prime}}\left(\frac{\omega_{1}+\omega_{2}}{2}, \frac{\mathbf{q}_{1}+\mathbf{q}_{2}}{2}\right) \delta\left(\omega-\omega_{1}+\omega_{2}\right) \delta\left(\mathbf{k}-\mathbf{q}_{1}+\mathbf{q}_{2}\right),
$$

the integrand in Eq. (28) reduces to

$$
\begin{aligned}
& W_{j j_{1}^{\prime} j_{2} j^{\prime}}\left(\mathbf{q}^{+}, \mathbf{q}_{1}^{+\prime}, \mathbf{q}_{1}^{-\prime}, \mathbf{q}^{-}\right) g_{j_{1}^{\prime} j_{2}}\left(q_{0}, \mathbf{q}_{1}^{\prime}\right)\left[G_{j^{\prime}}^{A}\left(q_{0}^{-}, \mathbf{q}^{-}\right)-G_{j}^{R}\left(q_{0}^{+}, \mathbf{q}^{+}\right)\right] \\
& +W_{j j_{1}^{\prime} j_{1}^{\prime} j_{2}}\left(\mathbf{q}^{+}, \mathbf{q}_{1}^{+^{\prime}}, \mathbf{q}_{1}^{+^{\prime}}, \mathbf{q}^{+}\right) g_{j_{2} j^{\prime}}\left(q_{0}, \mathbf{q}\right) G_{j_{1}^{\prime}}^{R}\left(q_{0}^{+}, \mathbf{q}_{1}^{+{ }^{\prime}}\right) \\
& -W_{j_{2} j_{1}^{\prime} j_{1}^{\prime} j^{\prime}}\left(\mathbf{q}^{-}, \mathbf{q}_{1}^{-{ }^{\prime}}, \mathbf{q}_{1}^{-^{\prime}}, \mathbf{q}^{-}\right) g_{j j_{2}}\left(q_{0}, \mathbf{q}\right) G_{j_{1}^{\prime}}^{A}\left(q_{0}^{-}, \mathbf{q}_{1}^{-{ }^{\prime}}\right),
\end{aligned}
$$

where $\mathbf{q}^{ \pm} \equiv \mathbf{q} \pm \mathbf{k} / 2, q_{0}^{ \pm} \equiv q_{0} \pm \omega / 2$, and $q_{0}$ is the temporal (frequency) component of the 3-vector $\left(q_{0}, \mathbf{q}\right)$.

The Green's functions $G^{A, R}$ (29) contain both singular ( $\delta$-type) and regular $[P(1 / x)]$ contributions. Under the usual circumstances, when the equation contains only the diagonal components of the Green's functions $g_{j j}$, the regular parts disappear from the differences $G_{j}^{A}-G_{j}^{R}$ and from the equation, and the Green's functions can be expressed via the deviation of the single-particle distribution from the equilibrium $\delta n_{j j}$ :

$$
g_{j j}(\omega, \mathbf{q})=2 \pi i \delta n_{j j}(\mathbf{q}) \delta\left(\omega-\epsilon_{j \mathbf{q}}+\mu\right) .
$$

Then the transport equation for the Green's function reduces to a standard Boltzmann-Waldmann-Snider equation (13). This is not the case when the equation contains the offdiagonal Green's functions $g_{j j^{\prime}}$, and the differences $G_{j}^{A}$ $-G_{j^{\prime}}^{R}$ retain the principal part integrals $P(1 / x)$.

\section{Coupling to off-diagonal Green's functions}

The coupling to off-diagonal Green's functions $\delta G_{j j^{\prime}}$ is important only when the separation between the energy levels $\Omega_{j j^{\prime}}$ is comparable to transition probabilities $W, \Omega_{j j^{\prime}}(j$ $\left.\neq j^{\prime}\right)=\left(\pi^{2} / 2 m L^{2}\right)\left|j^{2}-j^{\prime 2}\right| \sim W$, i.e., for not very thin films. If $\Omega_{j j^{\prime}}\left(j \neq j^{\prime}\right) \gg W$, one can neglect the off-diagonal $\delta G_{j j^{\prime}}$, and all the difficulties disappear. The opposite case $\Omega_{j j^{\prime}}(j$ $\left.\neq j^{\prime}\right) \ll W$ is effectively a single-band case and is also simple.

The coupling to off-diagonal components of the Green's function $\delta G_{j j^{\prime}}$ in Eq. (30) hinders the transition from the transport equation for the Green's function $g_{j j^{\prime}}$ to the equation for the distribution functions $\delta n_{j j^{\prime}}$ : the integration of the type (26) cannot transform simultaneously all the Green's functions $\delta G_{j j^{\prime}}$ in Eq. (30) into $\delta n_{j j^{\prime}}$. In other words, it is impossible to represent all $\delta G_{j j^{\prime}}$ in the form (31) so that all the $\delta$ functions in the equations get canceled out in the same uniform manner as in the case of purely diagonal equations.

At present, we can solve this problem for low-temperature degenerate systems $T \rightarrow 0$ by using the method that we have developed recently ${ }^{58}$ for the spin dynamics of the offdiagonal single-particle density matrix $n_{\uparrow \downarrow}$ in spin-polarized Fermi liquids. (The same approach to high-temperature systems is less constructive: it leads to an extra integral equation instead of a finite set of linear equations as in the case of degenerate systems.) The main difference from Ref. 58 is that Ref. 58 describes a two-level exchange system for which the interaction does not depend on the energy level and reduces to products of Kronecker $\delta$ s in level indices $j$. As a result, we performed the exact summation of all diagrams and calculated the Fermi liquid renormalizations to the transport equation. Our present multiband system with large indices $j$ requires inversion of large matrices. Since the interaction depends explicitly on the band indices $j$ and does not reduce to a product of Kronecker symbols in indices $j$, the explicit analytical inversion is impossible and the Fermiliquid renormalizations cannot be calculated.

In the anomalous region $\Omega_{j j^{\prime}} \sim W$, we can split $g_{j j^{\prime}}$ into regular $(R)$ and singular $(S)$ parts, $g_{j j^{\prime}}=g_{j j^{\prime}}^{R}+g_{j j^{\prime}}^{S}$, where the singular part contains the $\delta$-type factors $\delta\left(\omega-\epsilon_{j \mathbf{q}}+\mu\right)$. Equation (30) for $g_{j j^{\prime}}^{R}$ contains $g_{j j^{\prime}}^{S}$ only in the integrands,

$$
W_{j j_{1}^{\prime} j_{2} j^{\prime}}\left(\mathbf{q}^{+}, \mathbf{q}_{1}^{+}, \mathbf{q}_{1}^{-}, \mathbf{q}^{-}\right) g_{j_{1}^{\prime} j_{2}}^{S}\left(q_{0}, \mathbf{q}_{1}\right) P\left(\frac{1}{q_{0}^{-}-\epsilon_{j^{\prime} \mathbf{q}}^{-}+\mu}-\frac{1}{q_{0}^{+}-\epsilon_{j \mathbf{q}}^{+}+\mu}\right),
$$

where $\epsilon_{j \mathbf{q}}^{ \pm} \equiv \epsilon_{j}(\mathbf{q} \pm \mathbf{k} / 2)$. Here the difference of the principal parts $P(1 / x)$ is of the order of $\omega-\mathbf{k} \cdot \mathbf{q} / m-\Omega_{j j^{\prime}}$ and is, therefore, of the order of $W$. Then the whole integrand (32) is of the second order in $W$ and is small. Since most of the terms with $g_{j j^{\prime}}^{R}$ in the same equation are of the first order in $W$, this makes $g_{j j^{\prime}}^{R} \ll g_{j j^{\prime}}^{S}$. This, in turn, means that $g_{j j^{\prime}}^{R}$ can be neglected in the integrands (28) and (32) of the collision operator in the equation for $g_{j j^{\prime}}^{S}$ : 


$$
\begin{aligned}
& i \pi W_{j j_{1}^{\prime} j_{2} j^{\prime}}\left(\mathbf{q}^{+}, \mathbf{q}_{1}^{+^{\prime}}, \mathbf{q}_{1}^{-^{\prime}}, \mathbf{q}^{-}\right) g_{j_{1}^{\prime} j_{2}}^{S}\left(q_{0}, \mathbf{q}_{1}^{\prime}\right)\left[\delta\left(q_{0}^{-}-\boldsymbol{\epsilon}_{j^{\prime}}^{-} \mathbf{q}^{+\mu}\right)+\delta\left(q_{0}^{+}-\boldsymbol{\epsilon}_{j \mathbf{q}}^{+}+\mu\right)\right] \\
& \quad+W_{j j_{1}^{\prime} j_{1}^{\prime} j_{2}}\left(\mathbf{q}^{+}, \mathbf{q}_{1}^{+^{\prime}}, \mathbf{q}_{1}^{+^{\prime}}, \mathbf{q}^{+}\right) g_{j_{2} j^{\prime}}^{S}\left(q_{0}, \mathbf{q}\right) G_{j_{1}^{\prime}}^{R}\left(q_{0}^{+}, \mathbf{q}_{1}^{+^{\prime}}\right) \\
& \quad-W_{j_{2} j_{1}^{\prime} j_{1}^{\prime} j^{\prime}}\left(\mathbf{q}^{-}, \mathbf{q}_{1}^{-^{\prime}}, \mathbf{q}_{1}^{-^{\prime}}, \mathbf{q}^{-}\right) g_{j j_{2}}^{S}\left(q_{0}, \mathbf{q}\right) G_{j_{1}^{\prime}}^{A}\left(q_{0}^{-}, \mathbf{q}_{1}^{-{ }^{\prime}}\right) .
\end{aligned}
$$

Without the off-diagonal Green's functions $g_{j j^{\prime}}^{S}$, all the $\delta$ functions in each term would be the same, and Eq. (33) could be easily written on this single mass surface as an equation for $\delta n_{j j^{\prime}}$ using Eq. (31). With the off-diagonal Green's functions $g_{j j^{\prime}}^{S}$ in Eq. (33), the terms contain different $\delta$ functions. Thus $g_{j j^{\prime}}^{S}$ cannot be written consistently in the form (31) with a single $\delta$ function, but should contain a linear combination of all these $\delta$ functions incorporating, therefore, all relevant mass surfaces

$$
g_{j j^{\prime}}^{S}\left(q_{0}, \mathbf{q}\right)=2 i \pi \sum_{i}\left[\delta n_{j j^{\prime}}^{i+}\left(q_{0}, \mathbf{q}\right) \delta\left(q_{0}^{+}-\boldsymbol{\epsilon}_{i \mathbf{q}}^{+}+\mu\right)+\delta n_{j j^{\prime}}^{i-}\left(q_{0}, \mathbf{q}\right) \delta\left(q_{0}^{-}-\boldsymbol{\epsilon}_{i \mathbf{q}}^{-}+\mu\right)\right]
$$

with the "partial distributions" $\delta n_{j j^{\prime}}^{i \pm}(\mathbf{q}) \equiv \delta n_{j j^{\prime}}^{i}\left(\epsilon_{i \mathbf{q}}^{ \pm} \mp \omega / 2-\mu ; \mathbf{q}\right)$. The full distribution function is

$$
\delta n_{j j^{\prime}}(\omega, \mathbf{k} ; \mathbf{q})=\sum_{i}\left[\delta n_{j j^{\prime}}^{i+}\left(\epsilon_{i \mathbf{q}}^{+}-\omega / 2-\mu ; \mathbf{q}\right)+\delta n_{j j^{\prime}}^{i-}\left(\epsilon_{i \mathbf{q}}^{-}+\omega / 2-\mu ; \mathbf{q}\right)\right]
$$

Finally, the kinetic equation becomes a set of equations for partial distributions $\delta n_{j j^{\prime}}^{i \pm}$ :

$$
\left(\omega-\frac{\mathbf{k} \cdot \mathbf{q}}{m}-\Omega_{j j^{\prime}}\right) \delta n_{j j^{\prime}}^{i+}(\mathbf{q})=\sum_{j_{1}^{\prime} j_{2}} \int \frac{d^{2} q_{1}}{(2 \pi)^{2}} Q_{j j^{\prime}, j_{1}^{\prime} j_{2}}^{+}\left(\mathbf{k}, \mathbf{q} ; \mathbf{q}_{1}\right)
$$

where the integrand $Q_{j j^{\prime}, j_{1}^{\prime} j_{2}}^{+}\left(\mathbf{k}, \mathbf{q} ; \mathbf{q}_{1}\right)$ is equal to

$$
\begin{aligned}
& i \pi W_{j j_{1}^{\prime} j_{2} j^{\prime}}\left(\mathbf{q}^{+}, \mathbf{q}_{1}^{+}, \mathbf{q}_{1}^{-}, \mathbf{q}^{-}\right) \delta_{i j} \\
& \quad \times \sum_{l}\left[\delta n_{j_{1}^{\prime} j_{2}}^{l+}\left(\mathbf{q}_{1}\right) \delta\left(\epsilon_{i \mathbf{q}}^{+}-\epsilon_{l \mathbf{q}_{1}}^{+}\right)+\delta n_{j_{1}^{\prime} j_{2}}^{l-}\left(\mathbf{q}_{1}\right) \delta\left(\epsilon_{i \mathbf{q}}^{+}-\epsilon_{l \mathbf{q}_{1}}^{-}-\omega\right)\right] \\
& \quad+W_{j j_{1}^{\prime} j_{1}^{\prime} j_{2}}\left(\mathbf{q}^{+}, \mathbf{q}_{1}^{+}, \mathbf{q}_{1}^{+}, \mathbf{q}^{+}\right) \delta n_{j_{2} j^{\prime}}^{i+}(\mathbf{q}) G_{j_{1}^{\prime}}^{R}\left(\epsilon_{i \mathbf{q}}^{+}-\mu, \mathbf{q}_{1}^{+}\right)-W_{j_{2} j_{1}^{\prime} j_{1}^{\prime} j^{\prime}}\left(\mathbf{q}^{-}, \mathbf{q}_{1}^{-}, \mathbf{q}_{1}^{-}, \mathbf{q}^{-}\right) \delta n_{j j_{2}}^{i+}(\mathbf{q}) G_{j_{1}^{\prime}}^{A}\left(\epsilon_{i \mathbf{q}}^{+}-\mu-\omega, \mathbf{q}_{1}^{-}\right) .
\end{aligned}
$$

The equation for $\delta n_{j j^{\prime}}^{i-}\left(q_{0}, \mathbf{q}\right)=\left[\delta n_{j j^{\prime}}^{i+}\left(q_{0}, \mathbf{q}\right)\right]^{\dagger}$ is the Hermitian conjugate to Eq. (36) with $\mathbf{k} \rightarrow-\mathbf{k}, \omega \rightarrow-\omega$. This equation should be compared with a standard kinetic equation which involves only diagonal states and can be written as a set of equations for densities of particles $\delta n_{j}=\delta n_{j j}^{j}$ on each level:

$$
\left(\omega-\frac{\mathbf{k} \cdot \mathbf{q}}{m}\right) \delta n_{j}^{+}(\mathbf{q})=\sum_{j_{1}^{\prime}} \int \frac{d^{2} q_{1}}{(2 \pi)^{2}} Q_{j j_{1}^{\prime}}^{+}\left(\mathbf{k}, \mathbf{q} ; \mathbf{q}_{1}\right)
$$

with the integrand $Q_{j j_{1}^{\prime}}^{+}\left(\mathbf{k}, \mathbf{q} ; \mathbf{q}_{1}\right)$,

$$
\begin{aligned}
& i \pi W_{j j_{1}^{\prime} j_{1}^{\prime} j}\left(\mathbf{q}^{+}, \mathbf{q}_{1}^{+}, \mathbf{q}_{1}^{-}, \mathbf{q}^{-}\right)\left[\delta n_{j_{1}^{\prime}}^{+}\left(\mathbf{q}_{1}\right) \delta\left(\boldsymbol{\epsilon}_{j \mathbf{q}}^{+}-\epsilon_{j_{1}^{\prime} \mathbf{q}_{1}}^{+}\right)+\delta n_{j_{1}^{\prime}}^{-}\left(\mathbf{q}_{1}\right) \delta\left(\epsilon_{j \mathbf{q}}^{+}-\epsilon_{j_{1}^{\prime} \mathbf{q}_{1}}^{-}-\omega\right)\right] \\
& \quad+\delta n_{j}^{+}(\mathbf{q})\left[W_{j j_{1}^{\prime} j_{1}^{\prime} j}\left(\mathbf{q}^{+}, \mathbf{q}_{1}^{+}, \mathbf{q}_{1}^{+}, \mathbf{q}^{+}\right) G_{j_{1}^{\prime}}^{R}\left(\boldsymbol{\epsilon}_{j \mathbf{q}}^{+}-\mu, \mathbf{q}_{1}^{+}\right)-W_{j j_{1}^{\prime} j_{1}^{\prime} j}\left(\mathbf{q}^{-}, \mathbf{q}_{1}^{-}, \mathbf{q}_{1}^{-}, \mathbf{q}^{-}\right) G_{j_{1}^{\prime}}^{A}\left(\boldsymbol{\epsilon}_{j \mathbf{q}}^{+}-\mu-\omega, \mathbf{q}_{1}^{-}\right)\right] .
\end{aligned}
$$

\section{Transport equation}

Equations (36) and (37) are quantum kinetic equations with finite $\omega$ and $\mathbf{k}$. For transport problems, we should consider these equations in a quasiclassical (and hydrodynamic) limit for the motion along the film (the motion across the film remains quantized). In other words, we should expand these equations in small $\mathbf{k}$ and $\omega$, and, for transport calculations, put $\omega=\mathbf{k}=0$ in the collision integral $Q$. Then Eq. (36) reduces to

$$
\left(\omega-\frac{\mathbf{k} \cdot \mathbf{q}}{m}-\Omega_{j j^{\prime}}\right) \delta n_{j j^{\prime}}^{i}(\mathbf{q})=\sum_{j_{1}^{\prime} j_{2}} \int \frac{d^{2} q_{1}}{(2 \pi)^{2}} Q_{j j^{\prime}, j_{1}^{\prime} j_{2}}\left(\mathbf{q}, \mathbf{q}_{1}\right)
$$

with 


$$
\begin{aligned}
Q_{j j^{\prime}, j_{1}^{\prime} j_{2}}= & i \pi W_{j j_{1}^{\prime} j_{2} j^{\prime}}\left(\mathbf{q}, \mathbf{q}_{1}, \mathbf{q}_{1}, \mathbf{q}\right)\left(\delta_{i j}+\delta_{i j^{\prime}}\right) \sum_{l} \delta n_{j_{1}^{\prime} j_{2}}^{l}\left(\mathbf{q}_{1}\right) \delta\left[\boldsymbol{\epsilon}_{i}(\mathbf{q})-\boldsymbol{\epsilon}_{l}\left(\mathbf{q}_{1}\right)\right]+W_{j j_{1}^{\prime} j_{1}^{\prime} j_{2}}\left(\mathbf{q}, \mathbf{q}_{1}, \mathbf{q}_{1}, \mathbf{q}\right) \delta n_{j_{2} j^{\prime}}^{i}(\mathbf{q}) \\
& \times G_{j_{1}^{\prime}}^{R}\left[\boldsymbol{\epsilon}_{i}(\mathbf{q})-\mu, \mathbf{q}_{1}\right]-W_{j_{2} j_{1}^{\prime} j_{1}^{\prime} j^{\prime}}\left(\mathbf{q}, \mathbf{q}_{1}, \mathbf{q}_{1}, \mathbf{q}\right) \delta n_{j j_{2}}^{i}(\mathbf{q}) G_{j_{1}^{\prime}}^{A}\left[\boldsymbol{\epsilon}_{i}(\mathbf{q})-\mu, \mathbf{q}_{1}\right]
\end{aligned}
$$

and $\delta n_{j j^{\prime}}^{i+}=\delta n_{j j^{\prime}}^{i-}=\delta n_{j j^{\prime}}^{i} / 2$. In the same limit, the standard kinetic equation (37) reduces to

$$
\left(\omega-\frac{\mathbf{k} \cdot \mathbf{q}}{m}\right) \delta n_{j}(\mathbf{q})=\sum_{j_{1}^{\prime}} \int \frac{d^{2} q_{1}}{(2 \pi)^{2}} Q_{j j_{1}^{\prime}}\left(\mathbf{q}, \mathbf{q}_{1}\right)
$$

with

$$
Q_{j j_{1}^{\prime}}=W_{j j_{1}^{\prime} j_{1}^{\prime} j}\left(\mathbf{q}, \mathbf{q}_{1}, \mathbf{q}_{1}, \mathbf{q}\right)\left\{2 i \pi \delta n_{j_{1}^{\prime}}\left(\mathbf{q}_{1}\right) \delta\left(\boldsymbol{\epsilon}_{j \mathbf{q}}-\boldsymbol{\epsilon}_{j_{1}^{\prime} \mathbf{q}}\right)+\delta n_{j}(\mathbf{q})\left[G_{j_{1}^{\prime}}^{R}\left(\boldsymbol{\epsilon}_{j \mathbf{q}}-\mu, \mathbf{q}_{1}\right)-G_{j_{1}^{\prime}}^{A}\left(\boldsymbol{\epsilon}_{j \mathbf{q}}-\mu, \mathbf{q}_{1}\right)\right]\right\}
$$

In the latter equation, the principal part integrals from $G_{j_{1}^{\prime}}^{R}$ and $-G_{j_{1}^{\prime}}^{A}(29)$ cancel each other while the $\delta$-type contributions are the same, and we return to the same Waldmann-Snider equations (13) and (17) as the one that we used earlier: ${ }^{41,42,40}$

$$
\left(\omega-\frac{\mathbf{k} \cdot \mathbf{q}}{m}\right) \delta n_{j}(\mathbf{q})=2 i \pi \sum_{j^{\prime}} \int \frac{d^{2} q^{\prime}}{(2 \pi)^{2}} W_{j j^{\prime} j^{\prime} j}\left(\mathbf{q}, \mathbf{q}^{\prime}, \mathbf{q}^{\prime}, \mathbf{q}\right)\left[\delta n_{j^{\prime}}\left(\mathbf{q}^{\prime}\right)-\delta n_{j}(\mathbf{q})\right] \delta\left(\epsilon_{j}(\mathbf{q})-\epsilon_{j^{\prime}}\left(\mathbf{q}^{\prime}\right)\right) .
$$

The transport equation (38) is a set of integral equations in the partial densities $\delta n_{j j^{\prime}}^{i}$ which describe the Green's functions $\delta G_{j j^{\prime}}$ on a set of mass surfaces. This situation is typical for transport problems which involve the off-diagonal states. The main obstacle to solving this set is a rapid growth in a size of the set with increasing number $S$ of occupied minibands $\epsilon_{j}$. While the standard Waldmann-Snider transport equation (39) consists of $S$ integral equations for complex variables $\delta n_{j} \equiv \delta n_{j j}^{j}$, the number of coupled integral equations (38) for $\delta n_{j j^{\prime}}^{i}$ increases as $S^{3}$. This makes an exact solution for systems with a large number of bands, as in the Boltzmann temperature range, practically impossible. For degenerate Fermi systems, the situation is better: the energy $\delta$ functions $\delta\left(\epsilon_{j}(\mathbf{q})-\epsilon_{F}\right)$ automatically reduce the integral equations in $\mathbf{q}$ to a set of $S^{3}$ linear equations at $\mathbf{q}=\mathbf{q}_{j F}$ for a finite number of bands $S$. These equations can be solved explicitly even for a fairly large number of bands $S$.

Calculation of transport coefficients, such as diffusion, mobility, or conductivity $\sigma$, should be done using the transport Eq. (38) in which we can disregard the principal part integrals in $G_{j_{1}^{\prime}}^{R}, G_{j_{1}^{\prime}}^{A}$,

$$
\begin{gathered}
i \pi \sum_{j_{1}^{\prime} j_{2}} \int \frac{d^{2} q_{1}}{(2 \pi)^{2}}\left[\begin{array}{c}
W_{j j_{1}^{\prime} j_{2} j^{\prime}}\left(\mathbf{q}, \mathbf{q}_{1}, \mathbf{q}_{1}, \mathbf{q}\right)\left(\delta_{i j}+\delta_{i j^{\prime}}\right) \sum_{l} \delta n_{j_{1}^{\prime} j_{2}}^{l}\left(\mathbf{q}_{1}\right) \delta\left(\boldsymbol{\epsilon}_{i \mathbf{q}}-\boldsymbol{\epsilon}_{l \mathbf{q}_{1}}\right) \\
-\left[W_{j j_{1}^{\prime} j_{1}^{\prime} j_{2}}\left(\mathbf{q}, \mathbf{q}_{1}, \mathbf{q}_{1}, \mathbf{q}\right) \delta n_{j_{2} j^{\prime}}^{i}(\mathbf{q})+W_{j_{2} j_{1}^{\prime} j_{1}^{\prime} j^{\prime}}\left(\mathbf{q}, \mathbf{q}_{1}, \mathbf{q}_{1}, \mathbf{q}\right) \delta n_{j j_{2}}^{i}(\mathbf{q})\right] \\
\times \delta\left(\epsilon_{j \mathbf{q}}-\epsilon_{j_{1}^{\prime} \mathbf{q}_{1}}\right)
\end{array}\right] \\
=-\Omega_{j j^{\prime}} \delta n_{j j^{\prime}}^{i}(\mathbf{q})-\frac{i}{m} \mathbf{F} \cdot \mathbf{q} \delta_{i j} \delta_{i j^{\prime}} \delta\left(\boldsymbol{\epsilon}_{j \mathbf{q}}-\mu\right),
\end{gathered}
$$

where $\mathbf{F}$ is the external force (electric field) and $\mu$ is the chemical potential (Fermi energy). The disregarded integrals compensate neglected diagrams of the first order in $\hat{V}$, but with $\hat{V}$ expanded up to the second order in $\xi$. This compensation is similar to the cancellation of Fermi-liquid renormalizations for Larmor frequencies of spin waves at $\mathbf{k}=\mathbf{0}$.

In degenerate systems, we look for the solutions in the form of angular Fourier harmonics on the Fermi surfaces:

$$
\delta n_{j j^{\prime}}^{i}(\mathbf{q})=\delta n_{j j^{\prime}}^{i}(\theta) \delta\left(\epsilon_{i \mathbf{q}}-\mu\right) .
$$

Then all the energy $\delta$ functions in Eq. (41) get canceled:

$$
\begin{aligned}
& m \sum_{j_{1}^{\prime} j_{2}} \int \frac{d \theta_{1}}{4 \pi}\left[\begin{array}{c}
\sum_{l} W_{j j_{1}^{\prime} j_{2} j^{\prime}}\left(q_{i}, q_{l}, \cos \chi\right)\left(\delta_{i j}+\delta_{i j^{\prime}}\right) \delta n_{j_{1}^{\prime} j_{2}}^{l}\left(\theta_{1}\right) \\
-\left[W_{j j_{1}^{\prime} j_{1}^{\prime} j_{2}}\left(q_{i}, q_{j_{1}^{\prime}}, \cos \chi\right) \delta n_{j_{2} j^{\prime}}^{i}(\theta)+W_{j_{2} j_{1}^{\prime} j_{1}^{\prime} j^{\prime}}\left(q_{i}, q_{j_{1}^{\prime}}, \cos \chi\right) \delta n_{j j_{2}}^{i}(\theta)\right]
\end{array}\right] \\
& \quad=i \Omega_{j j^{\prime}} \delta n_{j j^{\prime}}^{i}(\theta)-F q_{j} \cos \theta \delta_{i j} \delta_{i j^{\prime}} / m
\end{aligned}
$$

where $q_{j}$ is the Fermi momentum on the level $j,\left[q_{j}^{2}+(\pi j / L)^{2}\right] / 2 m=\mu, W_{j j_{1}^{\prime} j_{2} j^{\prime}}\left(\mathbf{q}, \mathbf{q}_{1}, \mathbf{q}_{1}, \mathbf{q}\right)$ becomes $W_{j j_{1}^{\prime} j_{2} j^{\prime}}\left(q_{i}, q_{l}, \cos \chi\right)$ with $|\mathbf{q}|=q_{i}$ and $\left|\mathbf{q}_{1}\right|=q_{l}, \theta$ (or $\theta_{1}$ ) is the angle between $\mathbf{F}$ and $\mathbf{q}$ (or $\mathbf{q}_{1}$ ), and $\chi$ is the angle between $\mathbf{q}$ and $\mathbf{q}_{1}$.

Only the first angular Fourier harmonic $\delta n_{j j^{\prime}}^{i(1)}$ of the distribution (42), 


$$
\begin{aligned}
\delta n_{j j^{\prime}}^{i}(\theta) & =\frac{1}{2} \delta n_{j j^{\prime}}^{i(0)}+\sum_{s=1}^{\infty} \delta n_{j j^{\prime}}^{i(s)} \cos (s \theta) \\
W(\chi) & =\frac{1}{2} W^{(0)}+\sum_{s=1}^{\infty} W^{(s)} \cos (s \chi), \quad W^{(s)}=2 \int_{0}^{2 \pi} W(\chi) \cos (s \chi) \frac{d \chi}{2 \pi},
\end{aligned}
$$

contributes to conductivity,

$$
\sigma=\frac{e^{2}}{2 \pi F} \sum_{i, j} q_{i} \delta n_{j j}^{i(1)}
$$

The equation for this harmonic has the form

$$
\begin{aligned}
& \frac{m}{4} \sum_{j_{1}^{\prime} j_{2}}\left[\left(\delta_{i j}+\delta_{i j^{\prime}}\right) \sum_{l} W_{j j_{1}^{\prime} j_{2} j^{\prime}}^{(1)}\left(q_{i}, q_{l}\right) \delta n_{j_{1}^{\prime} j_{2}}^{l(1)}-\left[W_{j j_{1}^{\prime} j_{1}^{\prime} j_{2}}^{(0)}\left(q_{i}, q_{j_{1}^{\prime}}\right) \delta n_{j_{2} j^{\prime}}^{i(1)}+W_{j_{2} j_{1}^{\prime} j_{1}^{\prime} j^{\prime}}^{(0)}\left(q_{i}, q_{j_{1}^{\prime}}\right) \delta n_{j j_{2}}^{i(1)}\right]\right] \\
& \quad=i \Omega_{j j^{\prime}} \delta n_{j j^{\prime}}^{i(1)}-F q_{j} \delta_{i j} \delta_{i j^{\prime}} / m .
\end{aligned}
$$

If one uses the simplified transport equation (40), then the analogs of Eqs. (46) and (45) are the standard equations

$$
\begin{aligned}
-F q_{j} / m & =\frac{m}{2} \sum_{j^{\prime}}\left[W_{j j^{\prime}}^{(1)}\left(q_{j}, q_{j^{\prime}}\right) \delta n_{j^{\prime}}^{(1)}-W_{j j^{\prime}}^{(0)}\left(q_{j}, q_{j^{\prime}}\right) \delta n_{j}^{(1)}\right] \\
\sigma & =\frac{e^{2}}{2 \pi F} \sum_{j} q_{j} \delta n_{j}^{(1)}
\end{aligned}
$$

with $W_{j j^{\prime}}^{(\beta)}\left(q_{j}, q_{j^{\prime}}\right) \equiv W_{j j^{\prime} j^{\prime} j}^{(\beta)}\left(q_{j}, q_{j^{\prime}}\right), \delta n_{j} \equiv \delta n_{j j}^{j}$.

\section{E. Comparison with scattering by bulk imperfections}

Transport processes with scattering by bulk impurities almost never exhibit any anomalies related to the off-diagonal terms in $j$. This difference between bulk-defined and walldefined transport should be explained.

Wall-defined transport requires averaging of surface inhomogeneities along coordinates $y, z$ (11), which results in the 2D $\delta$ function $\delta\left(\mathbf{q}_{1}-\mathbf{q}_{2}^{\prime}\right)$, i.e., the average momentum conservation law along the walls. There is no averaging over coordinate $X$ described by discrete indices $j$. Therefore, the transition probabilities (11) $W_{j_{1} j_{1}^{\prime} j_{2} j_{2}^{\prime}}$ contain terms both with Kronecker symbols $\delta_{j_{1} j_{1}^{\prime}}$ or $\delta_{j_{2} j_{2}^{\prime}}$, originating from the terms in energy that do not depend on $X$ (a discrete analog of momentum conservation for $P_{x}$ ), and the off-diagonal terms originating from $X P_{x}$. The latter terms are responsible for all anomalies.

For bulk impurities, it is usually assumed that the distance between the impurities $r_{i} \ll L$. Then there is an additional averaging over the impurity distribution across the film which leads to an effective homogenization along $X$ and the momentum conservation law, i.e., to the $\delta$ function over the indices $j$,

$$
W_{j_{1} j_{1}^{\prime} j_{2} j_{2}^{\prime}}\left(\mathbf{q}_{1}, \mathbf{q}_{1}^{\prime}\right) \rightarrow N_{i} U_{j_{1} j_{1}^{\prime}}\left(\mathbf{q}_{1}-\mathbf{q}_{1}^{\prime}\right) U_{j_{2} j_{2}^{\prime}}\left(\mathbf{q}_{1}^{\prime}-\mathbf{q}_{1}\right) \delta_{j_{1} j_{2}^{\prime}},
$$

where $N_{i}$ is the concentration of the impurities and $U$ is the potential distortion caused by a single impurity. What is more, scattering processes by different impurities are often assumed to be independent, meaning that the particle wavelength $\lambda \ll r_{i} \ll L$. This, in turn, means that the motion across the film is quasiclassical, and the discrete indices for the motion across the film should be replaced by continuous conserved momenta $p_{x}$ :

$$
W_{j_{1} j_{1}^{\prime} j_{2} j_{2}^{\prime}}\left(\mathbf{q}_{1}, \mathbf{q}_{1}^{\prime}\right) \rightarrow N_{i} U\left(\mathbf{p}_{1}-\mathbf{p}_{1}^{\prime}\right) U\left(\mathbf{p}_{1}^{\prime}-\mathbf{p}_{1}\right) \delta_{j_{1} j_{2}^{\prime}}
$$

Then $\delta n_{j j^{\prime}}^{i}(\mathbf{q})$ are replaced by $\delta n(\mathbf{p}) \delta_{j j^{\prime}} \delta_{i j}$, and we recover a standard classical transport equation. We will discuss the quasiclassical limit for Eq. (38) in more detail in the next subsection.

If the number of bulk impurities on the thickness of the film is not large, there is no averaging over the coordinate $X$. In this case, one can neglect the off-diagonal terms in the transport equation only if the matrix $U_{j j^{\prime}}$ is diagonal. If $U_{j j^{\prime}}$ 
is not diagonal, one immediately encounters the same problems as in the preceding sections for scattering by rough walls, and the transport equation for thin films with impurities assumes the anomalous form (38). We did not find the analysis of this situation in the literature.

\section{F. Quasiclassical limit}

In general, one should be cautious when studying the quasiclassical limit of thick films for ballistic particles. There is always some constructive or destructive interference between direct and reflected particles depending on whether the quantum numbers $j$ are even or odd. Without bulk attenuation, this interference does not disappear irrespective of how thick is the film. Therefore, the situation is not "quasiclassical" in a rigorous sense, and there could be a residual oscillatory dependence on the level index even for thick films. This situation is analogous to the following well-known paradox in optics. Suppose one wants to evaluate optical reflection from a semi-infinite space by using the results on reflection from films in an infinitely thick film limit. To get the classical result, one should not only take the limit of very thick films, but should also introduce some infinitely small bulk attenuation. Without the attenuation, the results strongly depend on whether the film thickness is equal to an even or odd number of half wavelengths, and a unique thick film limit simply does not exist. In this paper we disregard bulk attenuation and some of the results for "quasiclassical" thick films retain residual quantum features of interwall interference.

The quasiclassical thick film limit $L \gg \lambda$ involves a large number of energy minibands $S \gg 1$ with a narrow spacing $\Omega_{j j^{\prime}}=\left(\pi^{2} / 2 m L^{2}\right)\left(j^{2}-j^{\prime 2}\right)$ and large quantum numbers, $j$ $\gg 1$. The interband transitions $j \leftrightarrow j^{\prime}$ are significant, according to Eqs. (10) and (11), only for $1 \sim\left|j-j^{\prime}\right| \ll j, j^{\prime}$. We introduce continuous variables as $\pi j / L \rightarrow p_{x}+k_{x} / 2, \pi j^{\prime} / L$ $\rightarrow p_{x}-k_{x} / 2$, and $\Omega_{j j^{\prime}}=p_{x} k_{x} / m$ with $k_{x} \ll p_{x}$. In the collision integral (30), we, as usual, neglect $\omega$ and $\mathbf{k}$ thus disregarding the gradient terms and replacing $g_{j j^{\prime}}$ for small $\left|j-j^{\prime}\right|$ by diagonal $g_{j j}$ :

$$
\begin{gathered}
\left(\omega-\frac{\mathbf{k} \cdot \mathbf{p}}{m}\right) g_{j j}=i \sum_{j^{\prime}} \int \frac{d^{2} q^{\prime}}{2 \pi} W_{j j^{\prime} j^{\prime} j}\left(\mathbf{q}, \mathbf{q}^{\prime}, \mathbf{q}^{\prime}, \mathbf{q}\right)\left[g_{j^{\prime} j^{\prime}}^{\prime} \delta\left(q_{0}-\boldsymbol{\epsilon}+\mu\right)-g_{j j} \delta\left(q_{0}-\boldsymbol{\epsilon}^{\prime}+\mu\right)\right], \\
g=g\left(q_{0}, \mathbf{q}\right), \quad g^{\prime}=g\left(q_{0}, \mathbf{q}^{\prime}\right), \quad \boldsymbol{\epsilon}=\boldsymbol{\epsilon}_{j \mathbf{q}}, \quad \boldsymbol{\epsilon}^{\prime}=\boldsymbol{\epsilon}_{j^{\prime} \mathbf{q}^{\prime}}
\end{gathered}
$$

(in the LHS, $\mathbf{p}$ and $\mathbf{k}$ are already the 3D vectors, while in the RHS we still have $2 \mathrm{D}$ vectors $\mathbf{q}$ and summation over discrete variable $j^{\prime}$ ). Now we can introduce the density Eq. (31):

$$
\left(\omega-\frac{\mathbf{k} \cdot \mathbf{p}}{m}\right) \delta n_{j}(\mathbf{q})=2 \pi i \sum_{j^{\prime}} \int \frac{d^{2} q^{\prime}}{(2 \pi)^{2}} W_{j j^{\prime} j^{\prime} j}\left(\mathbf{q}, \mathbf{q}^{\prime}, \mathbf{q}^{\prime}, \mathbf{q}\right)\left[\delta n_{j^{\prime}}\left(\mathbf{q}^{\prime}\right)-\delta n_{j}(\mathbf{q})\right] \delta\left(\boldsymbol{\epsilon}_{j \mathbf{q}}-\boldsymbol{\epsilon}_{j^{\prime} \mathbf{q}^{\prime}}\right) .
$$

In $W_{j j^{\prime} j^{\prime} j}\left(\mathbf{q}, \mathbf{q}^{\prime}, \mathbf{q}^{\prime}, \mathbf{q}\right)(15)$ we should keep only the terms (16) that do not vanish in combination with the energy $\delta$ function in the integrand,

$$
\begin{gathered}
\frac{\pi j}{L} \rightarrow p_{x}, \quad \delta n_{j}(\mathbf{q}) \rightarrow \delta n(\mathbf{p}), \\
W_{j j^{\prime} j^{\prime} j}\left(\mathbf{q}, \mathbf{q}^{\prime}\right) \rightarrow W\left(\mathbf{p}, \mathbf{p}^{\prime}\right)=\frac{p_{x}^{2} p_{x}^{\prime 2}}{m^{2} L^{2}}\left[\zeta_{11}\left(\mathbf{q}^{\prime}-\mathbf{q}\right)+\zeta_{22}\left(\mathbf{q}^{\prime}-\mathbf{q}\right)+2 \zeta_{12}\left(\mathbf{q}^{\prime}-\mathbf{q}\right)(-1)^{j+j^{\prime}}\right] .
\end{gathered}
$$

The last, oscillatory term with $(-1)^{j+j^{\prime}}$ violates the quasiclassical nature of this expression and describes the inherent interference for ballistic particles reflected from the opposite sides of the (thick) film which is mentioned in the beginning of this section. Without interwall correlation, $\zeta_{12}=0$, or when the averaging (summation) over $j^{\prime}$ makes this oscillatory term disappear, we get the truly quasiclassical transport equation

$$
\left(\omega-\frac{\mathbf{k} \cdot \mathbf{p}}{m}\right) \delta n(\mathbf{p})=\frac{2 \pi i}{m^{2} L} \int \frac{d^{3} p^{\prime}}{(2 \pi)^{3}} p_{x}^{2} p_{x}^{\prime 2}\left[\zeta_{11}\left(\mathbf{q}^{\prime}-\mathbf{q}\right)+\zeta_{22}\left(\mathbf{q}^{\prime}-\mathbf{q}\right)\right]\left[\delta n\left(\mathbf{p}^{\prime}\right)-\delta n(\mathbf{p})\right] \delta\left(\frac{p^{2}}{2 m}-\frac{p^{\prime 2}}{2 m}\right) .
$$

The same equation appears in the presence of small residual bulk attenuation, which makes the interwall correlations irrelevant for thick films.

In two cases, the complication caused by the oscillatory terms $(-1)^{j+j^{\prime}} \zeta_{12}$ can be circumvented. The core issue is the dependence of the correlation function $\zeta$ on the quantum numbers $j, j^{\prime}$. If the correlation function is a slow function of $j^{\prime}$, then the summation over $j^{\prime}$ in Eq. (49) with $W(50)$ makes the contribution from the rapidly oscillating interwall term with $(-1)^{j+j^{\prime}} \zeta_{12}$ small in comparison to the intrawall term $\zeta_{11}+\zeta_{22}$, and one recovers Eq. (51).

If the wall-induced interband transitions are suppressed in comparison with the intraband scattering, the dependence of the correlation function $\zeta$ on the quantum numbers $j, j^{\prime}$ reduces to $\delta_{j j^{\prime}}$. Then the correlation functions $\zeta_{i k}\left(\mathbf{q}^{\prime}-\mathbf{q}\right)$ effectively contain the $\delta$ functions $\delta\left(\mathbf{q}^{\prime}-\mathbf{q}\right)$, and the summation over $j^{\prime}$ becomes trivial: 


$$
\left(\omega-\frac{\mathbf{k} \cdot \mathbf{p}}{m}\right) \delta n(\mathbf{p})=\frac{2 \pi i}{m^{2} L} \int \frac{d^{3} p^{\prime}}{(2 \pi)^{3}} p_{x}^{2} p_{x}^{\prime 2}\left[\zeta_{11}\left(\mathbf{q}^{\prime}-\mathbf{q}\right)+\zeta_{22}\left(\mathbf{q}^{\prime}-\mathbf{q}\right)+2 \zeta_{12}\left(\mathbf{q}^{\prime}-\mathbf{q}\right)\right]\left[\delta n\left(\mathbf{p}^{\prime}\right)-\delta n(\mathbf{p})\right] \delta\left(\frac{p^{2}}{2 m}-\frac{p^{\prime 2}}{2 m}\right) .
$$

A simple "quasiclassical" extrapolation for the oscillating interwall term $(-1)^{j+j^{\prime}} \zeta_{12}$ is to replace it by $\zeta_{12} \cos \left[L\left(p_{x}\right.\right.$ $\left.-p_{x}^{\prime}\right)$ :

$$
\begin{aligned}
(\omega- & \left.\frac{\mathbf{k} \cdot \mathbf{p}}{m}\right) \delta n(\mathbf{p})=\frac{2 \pi i}{m^{2} L} \int \frac{d^{3} p^{\prime}}{(2 \pi)^{3}} p_{x}^{2} p_{x}^{\prime 2}\left[\delta n\left(\mathbf{p}^{\prime}\right)-\delta n(\mathbf{p})\right] \delta\left(\frac{p^{2}}{2 m}-\frac{p^{\prime 2}}{2 m}\right) \\
& \times\left\{\zeta_{11}\left(\mathbf{q}^{\prime}-\mathbf{q}\right)+\zeta_{22}\left(\mathbf{q}^{\prime}-\mathbf{q}\right)+2 \cos \left[L\left(p_{x}-p_{x}^{\prime}\right)\right] \zeta_{12}\left(\mathbf{q}^{\prime}-\mathbf{q}\right)\right\} .
\end{aligned}
$$

A more accurate quasiclassical approach to the oscillating term in Eq. (50) for the Gaussian correlation function in the whole range of parameters (especially when $R \sim L$ ) is virtually impossible. It is easy to see that transport beyond the limiting cases (51) and (52) is dominated by particles with low quantum numbers $j$ and the accuracy of the quasiclassical approach is relatively low anyway.

\section{GENERAL ANALYTICAL RESULTS FOR TRANSPORT COEFFICIENTS}

One of the difficulties in solving the transport equation is the large number of accessible minibands $S$ which results in a high rank of the matrix transport equation. The transport equation has simple analytical solutions in three limiting cases of narrow clearances, $R / L \gg 1$, single-band occupancy, and long-wave particles, $p_{F} R \sim R S / L \ll 1$. In these cases, the difference between the standard and anomalous equations (46) and (47) is insignificant, and one should analyze only Eq. (47). In the first case, the gaps between minibands $\epsilon_{j}$ are large in comparison to the wall-induced perturbation, and the collision-induced interband transitions are ineffective in comparison with the intraband scattering. This makes the matrix $W_{j j^{\prime}}$ diagonal and simplifies the oscillating interwall term in $W_{j j^{\prime}}(16)$. Then $\sigma$ is determined by the angular harmonics of the correlators $\zeta_{i k}\left(\mathbf{q}_{j}-\mathbf{q}_{j}^{\prime}\right)$ on the Fermi surfaces $\epsilon_{j \mathbf{q}}=\mu$ :

$$
\begin{gathered}
\sigma=\pi e^{2}\left(\frac{L}{\pi}\right)^{4} \sum_{j} \frac{\nu(z)-j^{2}}{j^{4}\left[\Upsilon_{j}^{(0)}-\Upsilon_{j}^{(1)}\right]}, \\
\Upsilon_{j}^{(0,1)} \equiv \Upsilon^{(0,1)}\left(z_{j}\right)=\zeta_{11}^{(0,1)}+\zeta_{22}^{(0,1)}+2 \zeta_{12}^{(0,1)} .
\end{gathered}
$$

The dependence on the interwall correlations $\zeta_{12}$ is trivial and remains the same even in the quasiclassical limit.

In the case of single-band occupancy, the matrix equation becomes scalar with the result the same as Eqs. (54) without summation:

$$
\sigma=\pi e^{2}\left(\frac{L}{\pi}\right)^{4} \frac{\nu(z)-1}{\left[\Upsilon_{1}^{(0)}-\Upsilon_{1}^{(1)}\right]}
$$

For the long-wave particles, the 2D scattering is almost specular irrespective of the details of the scattering potential (quantum reflection). All the scattering probabilities are constants with the first harmonic equal to zero, and the summation over $j^{\prime}$ yields

$$
\begin{gathered}
\sigma=\frac{3 e^{2} L^{4} / \pi^{3}}{S(S+1)(2 S+1)\left[\zeta_{11}(0)+\zeta_{22}(0)\right]} \sum_{j=1}^{S} \frac{\nu(z)-j^{2}}{j^{2}\left(1+\Xi_{j}\right)}, \\
\Xi_{j}=\frac{6}{2 S+1} \frac{(-1)^{j+S} \zeta_{12}(0)}{\zeta_{11}(0)+\zeta_{22}(0)}, \quad \zeta_{i k}(0) \equiv \zeta_{i k}(\mathbf{q}=0) .
\end{gathered}
$$

Since the terms with the smallest $j$ make the largest contribution to the sum, the contribution of the interwall term $\Xi$ is an oscillating function of the total number of the occupied miniband $S$. The interwall term $\Xi$ rapidly declines with the increase in $S$ and disappears in the quasiclassical limit $S$ $\gg 1$. The typical behavior of the conductivity $\sigma(56)$ at $p_{F} R$ $\sim R S / L \ll 1$ for moderate $S$ is illustrated in Sec. V D.

For the intermediate values of $R$, the contribution of interwall correlations should be studied numerically for particular forms of the correlation functions of surface inhomogeneities.

\section{TRANSPORT COEFFICIENTS FOR GAUSSIAN CORRELATIONS OF SURFACE INHOMOGENEITIES}

\section{A. Parametrization of equations}

In this section we supplement general expressions by analytical and numerical solutions for Gaussian correlations of surface inhomogeneities (3). For numerical calculations we need to rewrite the equations and matrix elements $W_{j j_{1}^{\prime} j_{2} j^{\prime}}^{(0,1)}$ in dimensionless variables similar to those used in Ref. 40.

The 2D density of spin- $\frac{1}{2}$ particles $N_{j}$ and the longitudinal Fermi momentum $q_{j}$ in each occupied miniband $j$ are given by the overall chemical potential (Fermi energy) $\mu=\epsilon_{F}$ as

$$
N_{j}=q_{j}^{2} / 2 \pi, \quad(\pi j / L)^{2}+q_{j}^{2}=2 m \mu,
$$

while $\mu$ is determined self-consistently by the total density of particles $N$,

$$
N=\sum_{j}^{S} N_{j}=m S \mu / \pi-\pi S(S+1)(2 S+1) / 12 L^{2},
$$


where $S$ is the number of occupied minibands. Instead of $\mu$, $q_{j}$, and $N_{j}$ we use dimensionless variables $\nu$ and $z_{j}$,

$$
\begin{gathered}
\nu=2 m \mu(L / \pi)^{2}, \quad z_{j}=2 N_{j} L^{2} / \pi=q_{j}^{2} L^{2} / \pi^{2}, \\
z \equiv \sum z_{j}=2 N L^{2} / \pi .
\end{gathered}
$$

Then Eq. (57) can be rewritten as

$$
z_{j}=\nu-j^{2}, \quad z=\nu S-S(S+1)(2 S+1) / 6 .
$$

All the minibands with the indices $j>S$ are empty, $z_{j}>S$ $=0$. The changes in number of occupied minibands $S$ $=1,2,3,4, \ldots$ occur at $z=Z_{S}, Z_{S}=S^{3}-S(S+1)(2 S+1) / 6$
$=0,3,13,34,70 \ldots$. The number of occupied levels $S$ for the given value of $z=2 N L^{2} / \pi$ is the integer part of $\nu^{1 / 2}(z)$,

$$
S=\operatorname{Int}[\sqrt{\nu}]=\operatorname{Int}[\sqrt{z / S+(S+1)(2 S+1) / 6}] .
$$

Computationally, it is more convenient to fix the number of bands $S$ and to determine the interval of the values of $z$ and $\nu$, which corresponds to this number of bands $S$.

The transport equation (46) with dimensionless distribution function $\kappa_{j j^{\prime}}^{i}$ and transition probabilities $\widetilde{W}_{j j_{1}^{\prime} j_{2} j^{\prime}}^{(0,1)}$,

$$
\begin{gathered}
\delta n_{j j^{\prime}}^{i(1)}=-\frac{F L^{3}}{\pi^{4} l^{2}} \kappa_{j j^{\prime}}^{i}, \\
W_{j j_{1}^{\prime} j_{2} j^{\prime}}^{(0,1)}\left(q_{i}, q_{l}\right)=\frac{2 \pi^{5} l^{2} R^{2}}{m^{2} L^{6}} \widetilde{W}_{j j_{1}^{\prime} j_{2} j^{\prime}}^{(0,1)}\left(z_{i}, z_{l}\right),
\end{gathered}
$$

reduces to the set of dimensionless linear equations

$$
\begin{aligned}
& \frac{R^{2}}{2 L^{2}} \sum_{j_{1}^{\prime}, j_{2}, l} \kappa_{j_{1}^{\prime} j_{2}}^{l}\left[\widetilde{W}_{j j_{1}^{\prime} j_{2} j^{\prime}}^{(1)}\left(z_{i}, z_{l}\right)\left(\delta_{i j}+\delta_{i j^{\prime}}\right)-\sum_{k} \delta_{i l}\left[\widetilde{W}_{j k k j_{1}^{\prime}}^{(0)}\left(z_{l}, z_{k}\right) \delta_{j_{2} j^{\prime}}+\widetilde{W}_{j_{2} k k j^{\prime}}^{(0)}\left(z_{l}, z_{k}\right) \delta_{j j_{1}^{\prime}}\right]\right] \\
& \quad=i \frac{L^{2}}{2 \pi^{3} l^{2}}\left(j^{2}-j^{\prime 2}\right) \kappa_{j j^{\prime}}^{i}+\sqrt{z_{j}} \delta_{i j} \delta_{i j^{\prime}}
\end{aligned}
$$

while the conductivity is described by the dimensionless function $\Pi(z, R / L)$,

$$
\sigma=\frac{2 e^{2} L^{2}}{\pi^{4} l^{2}} \Pi\left(z, \frac{R}{L}\right), \quad \Pi\left(z, \frac{R}{L}\right)=-\frac{1}{4} \sum_{i, j} \sqrt{z_{i}} \kappa_{j j}^{i}
$$

In the same notations, the standard transport equation (47) has the form

$$
\begin{gathered}
\sqrt{z_{j}}=\frac{R^{2}}{L^{2}} \sum_{j^{\prime}}\left[\widetilde{W}_{j j^{\prime} j^{\prime} j}^{(1)}\left(z_{j}, z_{j^{\prime}}\right) \kappa_{j^{\prime}}-\widetilde{W}_{j j^{\prime} j^{\prime} j}^{(0)}\left(z_{j}, z_{j^{\prime}}\right) \kappa_{j}\right], \quad \kappa_{j} \equiv \kappa_{j j}^{j}, \\
\sigma=\frac{2 e^{2} L^{2}}{\pi^{4} l^{2}} \tilde{\Pi}\left(z, \frac{R}{L}\right), \quad \tilde{\Pi}\left(z, \frac{R}{L}\right)=-\frac{1}{4} \sum_{j} \sqrt{z_{j}} \kappa_{j} .
\end{gathered}
$$

Further calculations require specific expressions for transition probabilities $\widetilde{W}(11),(16)$, and (62).

\section{B. Transition probabilities}

Though it is possible to perform the computation with all parameters in the correlation functions $\zeta_{i j}, \widetilde{\zeta}_{i j}$, it is worth limiting the number of independent parameters. We assume that all the intrawall and interwall correlation radii $R_{i k}$ are the same, while the average heights $l_{i j}$ of inhomogeneities are different, $l_{i k}^{2}=a_{i k} l^{2}$, though of the same scale $l$,

$$
\zeta_{i j}=2 \pi a_{i j} l^{2} R^{2} \exp \left[-\left(\mathbf{q}^{\prime}-\mathbf{q}\right)^{2} R^{2} / 2\right] .
$$

Then the harmonics $\widetilde{W}_{j j^{\prime} j^{\prime} j}^{(0,1)}\left(z_{j}, z_{j^{\prime}}\right)(11)$ in the simplified transport equation Eq. (65) are equal to

$$
\widetilde{W}_{j j^{\prime} j^{\prime} j}^{(0,1)}=\left[a_{11}+a_{22}+2 a_{12}(-1)^{j+j^{\prime}}\right] j^{2} j^{\prime 2} Q_{0}^{(0,1)}\left(z_{j} z_{j^{\prime}}\right) \text {. }
$$

The zeroth and first angular harmonics $Q_{0}^{(0,1)}$ of the exponent in the correlation function $\zeta_{i j}(66)$ are expressed via hypergeometric functions ${ }_{1} F_{1}$ in the same way as in Refs. 42 and 40:

$$
Q_{0}^{(0,1)}\left(z_{i}, z_{l}\right)=\exp \left[-\left(\sqrt{z_{i}}-\sqrt{z_{l}}\right)^{2} \frac{\pi^{2} R^{2}}{2 L^{2}}\right]\left[{ }_{1} F_{1}\left(\frac{1}{2}, 2,-2 \sqrt{z_{i} z_{l}} \frac{\pi^{2} R^{2}}{L^{2}}\right) \pm{ }_{1} F_{1}\left(\frac{3}{2}, 2,-2 \sqrt{z_{i} z_{l}} \frac{\pi^{2} R^{2}}{L^{2}}\right)\right],
$$

In the next subsection, we compare transport coefficients given by the full transport equation (63) and its simplified version (65). Since the full equation is already too cumbersome, we assume, for this particular task, that all normal and supplemental intrawall correlations $\zeta_{i i}, \widetilde{\zeta}_{i i}$ are the same, and that there are no interwall correlations:

$$
\zeta_{11}=\zeta_{22}=\widetilde{\zeta}_{11}=\widetilde{\zeta}_{22}=2 \pi l^{2} R^{2} \exp \left[-\left(\mathbf{q}^{\prime}-\mathbf{q}\right)^{2} R^{2} / 2\right], \quad \zeta_{12}=\widetilde{\zeta}_{12}=0
$$

[the interwall correlations are analyzed in Sec. V F]. Then the transition probabilities $\widetilde{W}_{j j_{1}^{\prime} j_{2} j^{\prime}}\left(z_{i}, z_{l}, \cos \chi\right)(11)$, 


$$
\begin{aligned}
\widetilde{W}_{j j_{1}^{\prime} j_{2} j^{\prime}}= & {\left[(-1)^{j+j_{1}^{\prime}+j_{2}+j^{\prime}}+1\right] \exp \left[-\left(z_{i}+z_{l}-2 \sqrt{z_{i} z_{l}} \cos \chi\right) \pi^{2} R^{2} / 2 L^{2}\right] } \\
& \times\left\{\left(j^{2} \delta_{j j_{1}^{\prime}}-\frac{1}{4} \frac{\left(i^{2}-l^{2}\right)^{2} \delta_{j j_{1}^{\prime}}}{z_{i}+z_{l}-2 \sqrt{z_{i} z_{l}} \cos \chi}-\left(i^{2}-l^{2}\right) \frac{\left(1-\delta_{j j_{1}^{\prime}}\right) j j_{1}^{\prime}}{j_{1}^{\prime 2}-j^{2}}\right)\right. \\
& \times\left(j_{2}^{2} \delta_{j_{2} j^{\prime}}-\frac{1}{4} \frac{\left(i^{2}-l^{2}\right)^{2} \delta_{j_{2} j^{\prime}}}{z_{i}+z_{l}-2 \sqrt{z_{i} z_{l}} \cos \chi}+\left(i^{2}-l^{2}\right) \frac{\left(1-\delta_{j_{2} j^{\prime}}\right) j_{2} j^{\prime}}{j^{\prime 2}-j_{2}^{2}}\right) \\
& \left.+\frac{1}{4} \frac{\left(i^{2}-l^{2}\right)^{2} z_{i} z_{l} \sin ^{2} \chi}{\left(z_{i}+z_{l}-2 \sqrt{z_{i} z_{l}} \cos \chi\right)^{2}} \delta_{j j_{1}^{\prime}} \delta_{j_{2} j^{\prime}}\right\},
\end{aligned}
$$

have the angular harmonics $\widetilde{W}_{j j_{1}^{\prime} j_{2} j^{\prime}}^{(0,1)}\left(z_{i}, z_{l}\right)$,

$$
\begin{aligned}
& \widetilde{W}_{j j_{1}^{\prime} j_{2} j^{\prime}}^{(\beta)}=\left[(-1)^{j+j_{1}^{\prime}+j_{2}+j^{\prime}}+1\right]\left(i^{2}-l^{2}\right)^{2} \\
& \times\left\{Q_{0}^{(\beta)}\left[\left(\frac{j^{2} \delta_{j j_{1}^{\prime}}}{i^{2}-l^{2}}-\frac{\left(1-\delta_{j j_{1}^{\prime}}\right) j j_{1}^{\prime}}{j_{1}^{\prime 2}-j^{2}}\right)\left(\frac{j_{2}^{2} \delta_{j_{2} j^{\prime}}}{i^{2}-l^{2}}+\frac{\left(1-\delta_{j_{2} j^{\prime}}\right) j_{2} j^{\prime}}{j^{\prime 2}-j_{2}^{2}}\right)-\frac{\delta_{j j_{1}^{\prime}} \delta_{j_{2} j^{\prime}}}{16}\right]\right. \\
& \left.-\frac{i^{2}-l^{2}}{4} Q_{1}^{(\beta)}\left[\delta_{j j_{1}^{\prime}} \frac{\left(1-\delta_{j_{2} j^{\prime}}\right) j_{2} j^{\prime}}{j^{\prime 2}-j_{2}^{2}}-\delta_{j_{2} j^{\prime}} \frac{\left(1-\delta_{j j_{1}^{\prime}}\right) j j_{1}^{\prime}}{j_{1}^{\prime 2}-j^{2}}+\frac{\delta_{j j_{1}^{\prime}} \delta_{j_{2} j^{\prime}}}{i^{2}-l^{2}}\left(j_{2}^{2}+j^{2}-\frac{z_{i}+z_{l}}{2}\right)\right]\right\} \text {, } \\
& Q_{\alpha}^{(\beta)}\left(z_{i}, z_{l}\right)=2 \int_{0}^{2 \pi} \frac{\exp \left[-\left(z_{i}+z_{l}-2 \sqrt{z_{i} z_{l}} \cos \chi\right) \pi^{2} R^{2} / 2 L^{2}\right]}{\left(z_{i}+z_{l}-2 \sqrt{z_{i} z_{l}} \cos \chi\right)^{\alpha}} \cos ^{\beta} \chi \frac{d \chi}{2 \pi}
\end{aligned}
$$

[both Greek indices $\alpha, \beta$ take the values $(0,1)]$. The hypergeometric integrals $Q_{0}^{(0,1)}$ are given by Eq. (68); integrals $Q_{1}$ cannot be expressed via special functions.

\section{Transport in the anomalous region}

This subsection illustrates the role of anomalous offdiagonal terms in the transport equation. We performed numerical calculations for conductivity on the basis of the full transport equation (63) and (64) and the standard equation (65) for the same values of parameters. We used the transition probabilities $\widetilde{W}$ with the Gaussian correlation of surface inhomogeneities (3) assuming that all the intrawall correlators are the same for both walls, and that there are no interwall correlations. For the full transport equation we used the transition probabilities (70), and for the simplified equation we used Eq. (67) with $a_{11}=a_{22}=1, a_{12}=0$.

Let us specify the range of parameters where one should expect noticeable off-diagonal contributions. The offdiagonal terms should be retained when $\Omega_{j j^{\prime}} \sim W$, i.e., according to Eq. (68), when

$$
1 \sim \frac{l^{2} R^{2} j^{3}}{L^{4}} \frac{1}{1+(R / L)^{3}\left(S^{2}-j^{2}\right)^{3 / 2}},
$$

where we disregarded all numerical factors and approximated ${ }_{1} F_{1}(3 / 2,2,-x)$ as $1 /\left[1+x^{3 / 2}\right]$. The difference $S^{2}$ $-j^{2}$ is either of the order of $S^{2}$ or of the order of $S$. If $S^{2}$ $-j^{2} \sim S^{2}$, then Eq. (72) is always violated, $\Omega_{j j^{\prime}} \gg W$, and we return to the simplified transport equation. Equation (72) can be valid only for

$$
S^{2}-j^{2} \sim S
$$

and either for

$$
S>\frac{L^{2}}{R^{2}}, \quad \frac{l^{2}}{L R} S^{3 / 2} \sim 1
$$

or for

$$
S<\frac{L^{2}}{R^{2}}, \quad \frac{l R}{L^{2}} S^{3 / 2} \sim 1 .
$$

Only then is there a noticeable difference between the full and simplified transport equation.

The computations with large values of $S$ are very difficult since the number of equations (63) grows as $2 S^{3}$. To see the off-diagonal contributions at moderate $S$, we should consider noticeable amplitudes $l$, though within the limits of the perturbation theory (8), $l \ll L, R$. The conditions (74) and (75) break down both at small $S \sim 1$ and at very large values of $S$. Since the number of occupied bands $S$ is a monotonic function of the density of particles $N$ (or dimensionless variable $z)$, the difference between the full and simplified transport equations can be observed only at intermediate values of $S$ (or $z$ ).

This effect is illustrated in Fig. 2 for $a_{11}=a_{22}=1, a_{12}$ $=0, R / L=0.5$, and the amplitude of inhomogeneities $l / R$ $=0.2$. For these values of $R$ and $L$, the largest deviation of the full function $\Pi(z, R / L)$ (64) (bold line in Fig. 2) from the function $\widetilde{\Pi}(z, R / L)(65)$ calculated from a standard transport equation without off-diagonal terms (dotted line) should be observed at $S \approx 13$ when the number of equations exceeds 4000. After that, the two curves should start to converge again. Since all the coefficients in the equations are compli- 


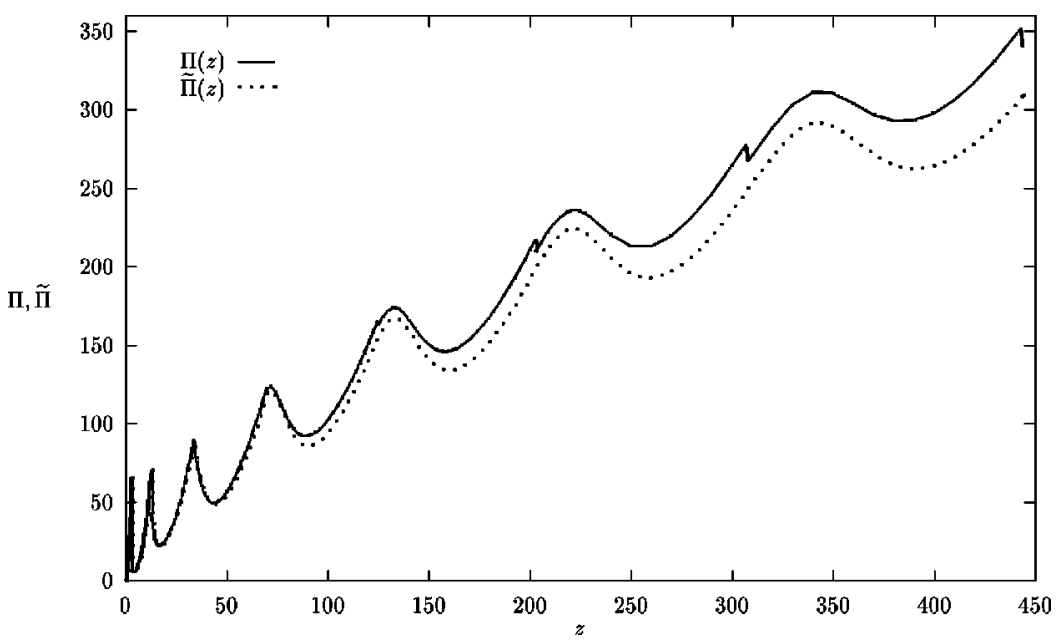

FIG. 2. $\Pi(z, R / L)$, Eq. (64) (solid line) and $\widetilde{\Pi}(z, R / L)$, Eq. (65) (dotted line), as a function of particle density $z=2 N L^{2} / \pi$ for $R / L$ $=0.5$ and $R / l=5, a_{11}=a_{22}=1, a_{12}=0$.

cated hypergeometric integrals, memory limitations allowed computation only up to $S=10$ when the anomalous offdiagonal contribution exceeded $10 \%$. To shift the maximal effect to smaller values of $S$, one would have to perform computations for inhomogeneities with larger $l$. This, in turn, would lower the accuracy of all expressions.

Small spikes on the bold line correspond to filling of higher energy bands with the increase in the number of particles $z$. It is not clear why the solution of the simplified transport equation does not exhibit these singularities for the same set of parameters. In general, our analysis of the simplified equation in Ref. 40 showed that the acuteness of these singularities strongly depends on the value $R / L$ (see below).

Figure 2 demonstrates that the conductivity in the anomalous regions (74) and (75) differs significantly from its value given by the standard transport equation. The data in Fig. 2 are the first exact transport computations for the anomalous region.

\section{Analytical results: Quantized motion}

In this subsection we present analytical results for the conductivity for Gaussian correlation of surface inhomogeneities outside the anomalous regions (74) and (75) in the same limiting cases as in Sec. IV B, namely, when $R / L \gg 1$ or $p_{F} R \ll 1$ for an arbitrary number of occupied bands $S$ [the Fermi momentum $\left.p_{F}^{2}=2 \pi N / S+(\pi / L)^{2}(S+1)(2 S+1) / 6\right]$ and for the case of single-band occupancy.

In the first case, $R / L \gg 1$, both walls are very close to each other, and multiple scattering occurs within the same inhomogeneity. In this case $z_{j}\left(\pi^{2} R^{2} / 2 L^{2}\right) \gg 1$ (except, maybe, for the highest miniband in which the number of particles $z_{S}$ can be small) and the exponents $\exp \left[-\left(\pi^{2} R^{2} / 2 L^{2}\right)\left(\sqrt{z_{j}}\right.\right.$ $\left.\left.-\sqrt{z_{j^{\prime}}}\right)^{2}\right]$ in functions $Q_{0}^{(0,1)}(68)$ are negligible for all $j$ $\neq j^{\prime}$ and can be replaced by the Kronecker symbol $\delta_{j j^{\prime}}$. This means that the interband transitions are effectively suppressed, and the set of equations (65) is diagonal:

$$
\kappa_{j}=-\frac{L^{2} \sqrt{z_{j}}}{2 R^{2} j^{4}\left(a_{11}+a_{22}+2 a_{12}\right)_{1} F_{1}\left[3 / 2,2,-2 \pi^{2} R^{2} z_{j} / L^{2}\right]} .
$$

Then the conductivity (65) is equal to

$$
\sigma=\frac{2 e^{2} L^{2}}{\pi^{4} l^{2}} \tilde{\Pi}(z, R / L), \quad \widetilde{\Pi}(z, R / L)=\frac{L^{2}}{8 R^{2}\left(a_{11}+a_{22}+2 a_{12}\right)} \sum_{j=1}^{S(z)} \frac{z_{j}}{{ }_{1} F_{1}\left[3 / 2,2,-2 \pi^{2} R^{2} z_{j} / L^{2}\right] j^{4}} .
$$

For all terms in the sum, except maybe the last one, for which $z_{S}$ may be small, the argument of the hypergeometric function is large, $2 \pi^{2} R^{2} z_{j} / L^{2} \gg 1$. Since

$$
x^{2} \gg 1, \quad{ }_{1} F_{1}\left((2 n-1) / 2, n,-x^{2}\right) \simeq \frac{(n-1) !}{\sqrt{\pi} x^{2 n-1}},
$$

the conductivity (77) reduces to [cf. Eq. (54)]

$$
\widetilde{\Pi}(z, R / L)=\frac{1}{a_{11}+a_{22}+2 a_{12}}\left[\frac{\pi^{7 / 2}}{2^{3 / 2}} \frac{R}{L} \sum_{j=1}^{S(z)-1} \frac{\left[\nu(z)-j^{2}\right]^{5 / 2}}{j^{4}}+\frac{L^{2}}{8 R^{2}} \frac{z_{S}}{{ }_{1} F_{1}\left[\frac{3}{2}, 2,-2 \pi^{2} R^{2} z_{S} / L^{2}\right] S^{4}}\right] .
$$




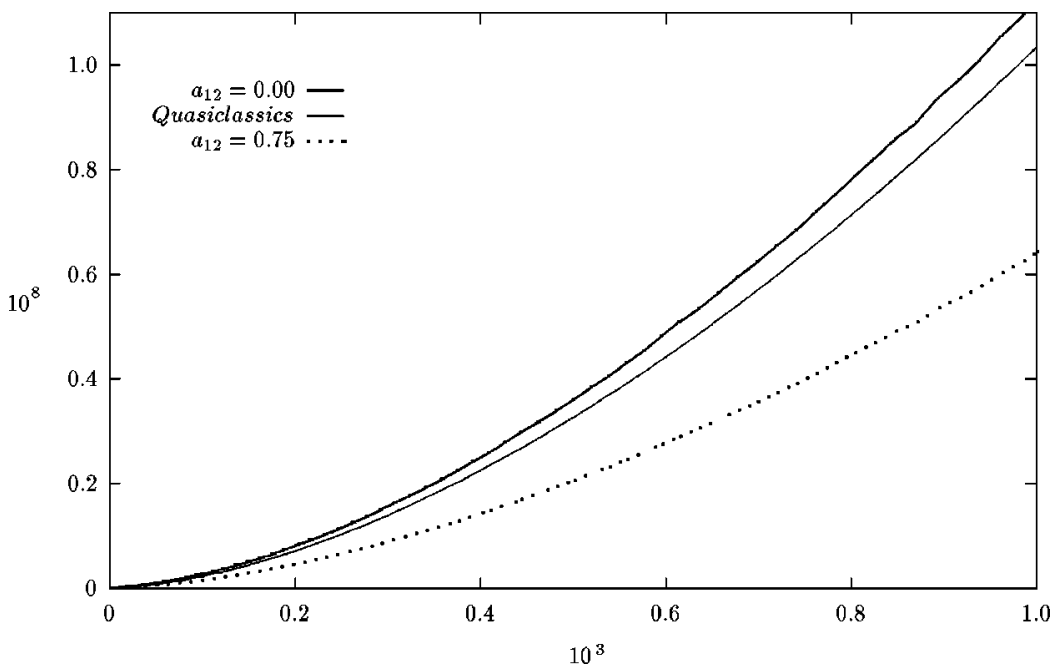

FIG. 3. $\widetilde{\Pi}(z, R / L)$, Eq. (65), as a function of particle density $z=2 N L^{2} / \pi$ for $R / L=50, a_{11}=a_{22}=1$. Solid line: $a_{12}=0$ (quantum); dotted line: $a_{12}=0.75$ (quantum); thin line: $a_{12}=0$ (quasiclassical).

Even for not very large $S$, the second term in the square brackets is small in comparison with the first one, while the sum itself converges faster than the sum $\Sigma 1 / j^{4}=\pi^{4} / 90 \simeq 1.082$ and is squeezed between $(\nu-1)^{5 / 2}$ and $1.082(\nu-1)^{5 / 2}$. Therefore, it is sufficient to leave in this equation only the first few terms in the sum,

$$
\widetilde{\Pi}(z, R / L) \simeq \frac{1}{a_{11}+a_{22}+2 a_{12}} \frac{\pi^{7 / 2}}{2^{3 / 2}} \frac{R}{L}\left[[\nu(z)-1]^{5 / 2}+\frac{[\nu(z)-4]^{5 / 2}}{16}+\frac{[\nu(z)-9]^{5 / 2}}{81}+\cdots\right] .
$$

This $\tilde{\Pi}(z, R / L)$ is a smooth function of the number of particles $z=2 N L^{2} / \pi$, and the singularities in the points where $S$ changes by 1 [an appearance of a new term in the sum (80)] are not noticeable.

In the case of single-band occupancy (55), the general result for conductivity is similar to Eqs. (77):

$$
\sigma=\frac{2 e^{2} L^{2}}{\pi^{4} l^{2}} \tilde{\Pi}(z, R / L), \quad \tilde{\Pi}(z, R / L)=\frac{L^{2}}{8 R^{2}\left(a_{11}+a_{22}+2 a_{12}\right)} \frac{z_{1}}{{ }_{1} F_{1}\left[3 / 2,2,-2 \pi^{2} R^{2} z_{1} / L^{2}\right]} .
$$

The function $\tilde{\Pi}(z, R / L)(79)$ is plotted in Fig. 3 for $R / L=50, a_{11}=a_{22}=1$, and two values of $a_{12}, a_{12}=0$ (thick line) and $a_{12}=0.75$ (dotted line). The intermediate line presents the quasiclassical results for $a_{12}=0$ and the same value $R / L$. All the curves, in accordance with Eq. (79), are simple monotonic functions of the number of particles $z$; the effect of interwall correlations $\left[a_{12}\right.$ in the denominators $\left.(77)-(80)\right]$ is noticeable, though trivial.

In the second limiting case $p_{F} R \ll 1$, the parameters $R \sqrt{z_{j}} / L \ll 1$, and all the hypergeometric functions in coefficients $Q$ (68) are equal to 1 . Then the set of transport equations (65) reduces to

$$
\sqrt{z_{j}}=-\frac{2 R^{2} j^{2}}{L^{2}} \kappa_{j} \sum_{j^{\prime}=1}^{S(z)} j^{\prime 2}\left[a_{11}+a_{22}+2 a_{12}(-1)^{j+j^{\prime}}\right] .
$$

After trivial summation, we get

$$
\kappa_{j}=-\frac{3 L^{2} \sqrt{z_{j}}}{R^{2} S(S+1) j^{2}\left[\left(a_{11}+a_{22}\right)(2 S+1)+6 a_{12}(-1)^{j+S}\right]}
$$

and the dimensionless conductivity (65) becomes [cf. Eq. (56)]

$$
\begin{gathered}
\tilde{\Pi}\left(z, \frac{R}{L}\right)=\frac{3 L^{2} / 4 R^{2}}{S(z)[S(z)+1][2 S(z)+1]} \frac{1}{a_{11}+a_{22}} \sum_{j=1}^{S(z)} \frac{\nu(z)-j^{2}}{j^{2}\left[1+\Xi_{j}\right]}, \\
\Xi_{j}=\frac{6}{2 S+1} \frac{(-1)^{j+S} a_{12}}{a_{11}+a_{22}} .
\end{gathered}
$$




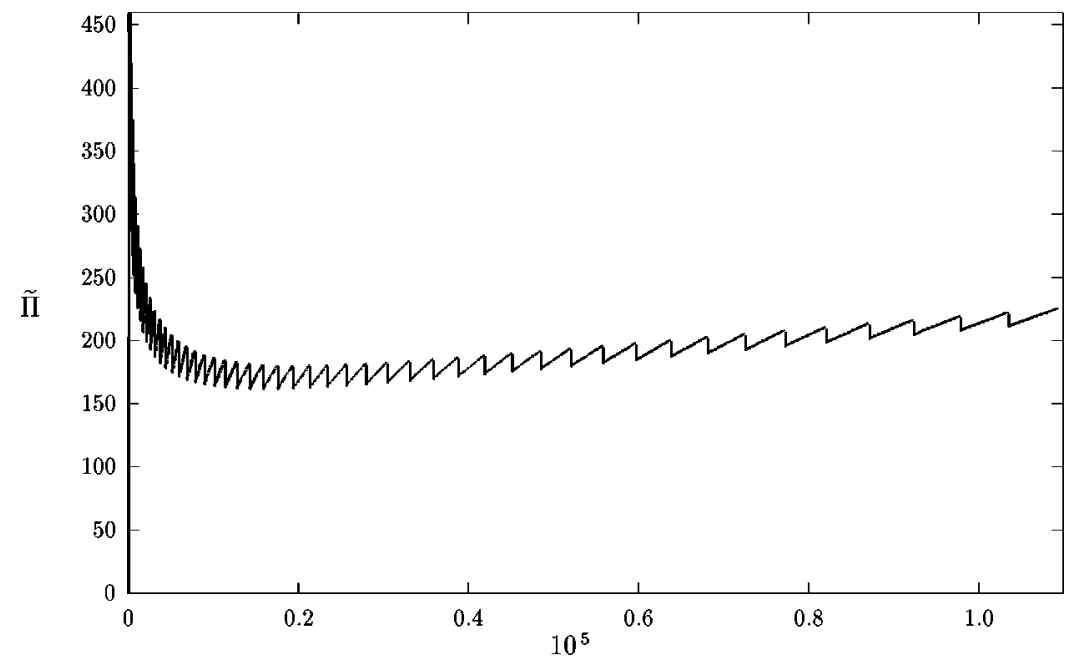

FIG. 4. $\widetilde{\Pi}(z, R / L)$, Eq. (65) as a function of particle density $z=2 N L^{2} / \pi$ for $R / L=0.01, a_{11}=a_{22}=1$ The results for $a_{12}=0 ; 0.75$ are indistinguishable.

This function is plotted in Figs. 4 (large values of $S$ ) and 5 (small $S$ ) for $R / L=0.001, a_{11}=a_{22}=1$, and two values of the interwall amplitude $a_{12}=0$ (solid line) and $a_{12}=0.75$ (dotted line). Figure 5 presents the blow-up of the initial part of Fig. 4 for small numbers of particles $z$ (small number of occupied states $S$ ) on a different scale.

Equation (84) contains the number of filled levels $S(z)$ explicitly in the denominator. In the points $z=Z_{S}=S^{3}$ $-S(S+1)(2 S+1) / 6$ the number of levels $S$ increases by $\Delta S=1$ and we see sharp drops in $\widetilde{\Pi}(z, R / L)$. The relative amplitude of these singularities should decrease with increasing $S$ as $1 / S$. Also, with increasing $S, \widetilde{\Pi}$ (84) becomes less and less dependent on the interwall term with $a_{12}$ and the solid and dotted curves in Fig. 4 (large $S$ ) merge. The difference between these two curves at small $S$ (Fig. 5) is quite large. Later, we will discuss the effect of interwall correlations in more detail.
Equation (84) for $\widetilde{\Pi}(z, R / L)$ can be simplified at $S \gg 1$ and $Z_{s} \approx 2 S^{3} / 3$ as

$$
\begin{aligned}
& \widetilde{\Pi}\left(z, \frac{R}{L}\right)=\frac{\pi^{2} L^{2}\left[1+\left(z-Z_{S}\right) / S^{3}(z)\right]}{16 R^{2}\left(a_{11}+a_{22}\right) S(z)}, \\
& \sigma=\frac{1+\left(z-Z_{S}\right) / S^{3}(z)}{8 \pi^{2} S(z)} \frac{e^{2} L^{4}}{l^{2} R^{2}} \frac{1}{a_{11}+a_{22}} .
\end{aligned}
$$

This function still contains sawlike singularities in the points $z=Z_{S}$ where $S(z)$ increases by 1 .

\section{E. Analytical results: Quasiclassical motion}

In the case of Gaussian correlations (66), the quasiclassical transport equation (51), (52) has the form

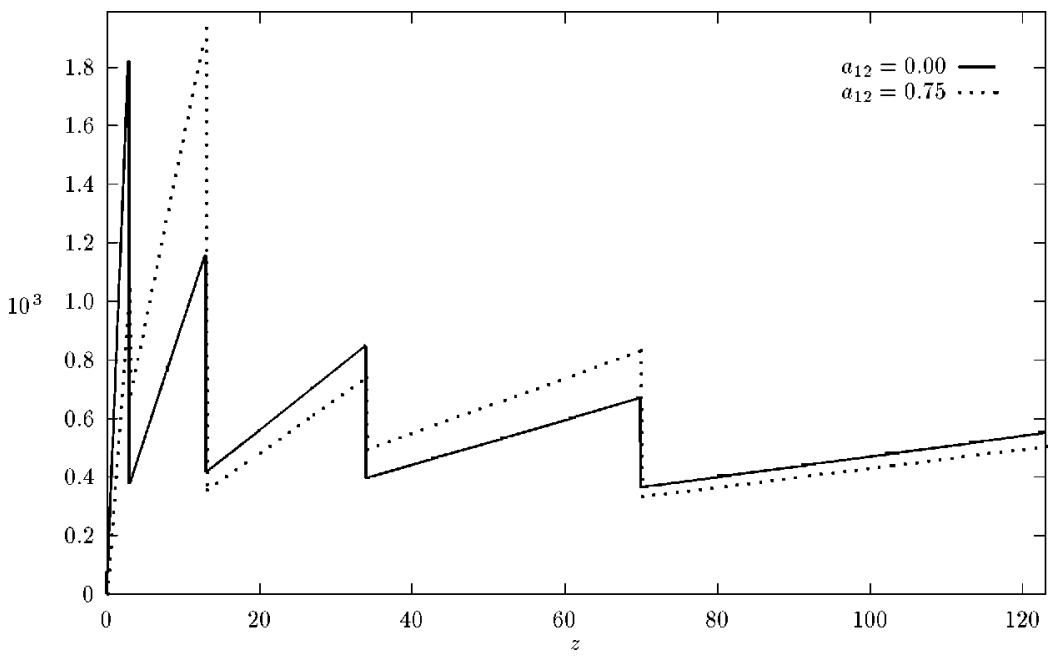

FIG. 5. Initial part of the curve from Fig. 4. Solid line: $a_{12}=0$; dotted line: $a_{12}=0.75$. 


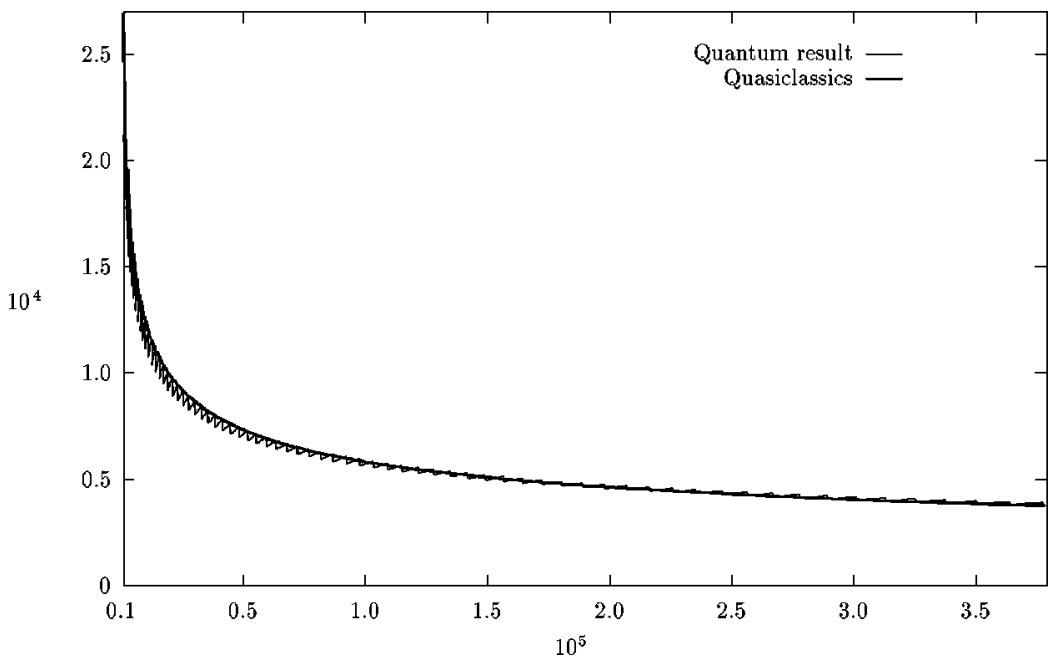

FIG. 6. $\widetilde{\Pi}(z, R / L)$, Eq. (65), as a function of particle density $z=2 N L^{2} / \pi$ for $R / L=0.001, a_{11}=a_{22}=1, a_{12}=0$. Smooth line, quasiclassical calculation (89); sawlike curve, quantum calculation (85).

$$
\frac{L^{2}}{R^{2}} \frac{\sqrt{z(\eta)}}{\{\hat{a}\}}=\int_{0}^{\sqrt{\nu}} d \eta^{\prime} \eta^{2} \eta^{\prime 2} \exp \left[-\frac{\pi^{2} R^{2}}{2 L^{2}}\left[\sqrt{z(\eta)}-\sqrt{z\left(\eta^{\prime}\right)}\right]^{2}\right]\left[\begin{array}{c}
{\left[\kappa\left(\eta^{\prime}\right)-\kappa(\eta)\right]_{1} F_{1}\left(\frac{1}{2} ; 2 ;-2 \pi^{2} \frac{R^{2}}{L^{2}} \sqrt{z(\eta) z\left(\eta^{\prime}\right)}\right)} \\
-\left[\kappa\left(\eta^{\prime}\right)+\kappa(\eta)\right]_{1} F_{1}\left(\frac{3}{2} ; 2 ;-2 \pi^{2} \frac{R^{2}}{L^{2}} \sqrt{z(\eta) z\left(\eta^{\prime}\right)}\right)
\end{array}\right],
$$

where $\{\hat{a}\}$ is an appropriate combination of amplitudes $a_{i k}$ from Eqs. (51) and (52), $\eta=p L / \pi$ is the dimensionless momentum, the number of particles $z(\eta)=\nu-\eta^{2}$ with momentum $\eta$ is the quasiclassical analog of the discrete $z_{j}$ $=2 N_{j} L^{2} / \pi$, and $\kappa(\eta)=-\pi^{4} l^{2} \delta n(\eta) / F L^{3}$ describes the deviation of the distribution function from equilibrium. In these notations, the conductivity is

$$
\sigma=\frac{2 e^{2} L^{2}}{\pi^{4} l^{2}} \tilde{\Pi}\left(p_{F} L, R / L\right), \quad \tilde{\Pi}=-\frac{1}{4} \int_{0}^{\sqrt{\nu}} d \eta \kappa(\eta) \sqrt{z(\eta)} .
$$

In the limiting case $p_{F} R \sim \pi R \sqrt{\nu} / L \ll 1$ and $S \sim \sqrt{\nu} \gg 1$, as in the corresponding quantum case, the interwall correlations vanish from the equation, and the proper combination of amplitudes is $\{\hat{a}\}=a_{11}+a_{22}$. The arguments $2 \pi^{2} R^{2} \sqrt{z(\eta) z\left(\eta^{\prime}\right)} / L^{2}$ of the hypergeometric functions are small, ${ }_{1} F_{1}\left(\frac{1}{2} ; 2 ; 0\right) \approx{ }_{1} F_{1}\left(\frac{3}{2} ; 2 ; 0\right) \approx 1$, and

$$
\kappa(\eta)=-\frac{3 L^{2}}{2 R^{2}} \frac{1}{a_{11}+a_{22}} \frac{\sqrt{z(\eta)}}{\nu^{3 / 2} \eta^{2}} .
$$

The integral (87) for the function $\widetilde{\Pi}\left(p_{F} L, R / L\right)$ with $\kappa(\eta)$ (88) diverges at small $p$ as $1 / p$. This divergence is natural: there is always a small number of particles with momenta parallel to the walls and, therefore, infinite mean free paths. This leads to a formal divergence of the integrand at $p_{x}$ $\rightarrow 0$ and requires a cutoff at small $p_{x}$. The cutoff parameter is determined either by bulk scattering or, in our case of ballistic particles without bulk attenuation, by the minimum momentum $p_{x} \sim \pi / L$. Since this is a quantum cutoff, the exact proportionality coefficient between the cutoff value $\left(p_{x}\right)_{\min }$ and $\pi / L$ cannot be guessed from the quasiclassical equations. We can either omit this coefficient or take it from the comparison with the exact quantum result $(85), \eta_{\min }$ $=6 / \pi^{2}$ :

$$
\sigma=\frac{2 e^{2} L^{2}}{\pi^{4} l^{2}} \tilde{\Pi}\left(p_{F} R, R / L\right)=\frac{\pi^{3}}{8} \frac{e^{2} L^{2}}{\pi^{4} l^{2}} \frac{L}{p_{F} R^{2}} \frac{1}{a_{11}+a_{22}} .
$$

This curve is presented as a bold line in Fig. 6 for $R / L$ $=10^{-3}$. The sawlike thin line presents the exact quantum expression (85). The highest values of $z$ in this figure correspond to more than a hundred occupied bands. At even higher values of the number of particles $z$, the inequality $p_{F} R \sim R S / L \ll 1$ will be violated, and the quasiclassical and quantum curves will diverge.

In the opposite limiting case, $R / L \gg 1$ and $S \gg 1$, the exponents $\exp \left[\left(-\pi^{2} R^{2} / 2 L^{2}\right)\left(\sqrt{z_{j}}-\sqrt{z_{j^{\prime}}}\right)^{2}\right]$ with $j \neq j^{\prime}$ in Eq. (86) should be neglected in comparison with the ones with $j=j^{\prime}$. The same is true for hypergeometric functions. Then the only important terms with $(-1)^{j+j^{\prime}}$ in Eqs. (51) and (52) are equal to 1 , and the proper combination of the amplitudes is $\{\hat{a}\}=a_{11}+a_{22}+2 a_{12}$. The solution of the transport equation has the form

$$
\kappa(\eta)=-\sqrt{2} \pi^{7 / 2} \frac{R}{L} \frac{1}{a_{11}+a_{22}+2 a_{12}} \frac{z^{2}(\eta)}{\eta^{4}} .
$$

The integral (87) also diverges at small $p$ as $1 / p^{3}$. The quantum cutoff $\eta_{\min }=\left(30 / \pi^{4}\right)^{1 / 3}$ yields 


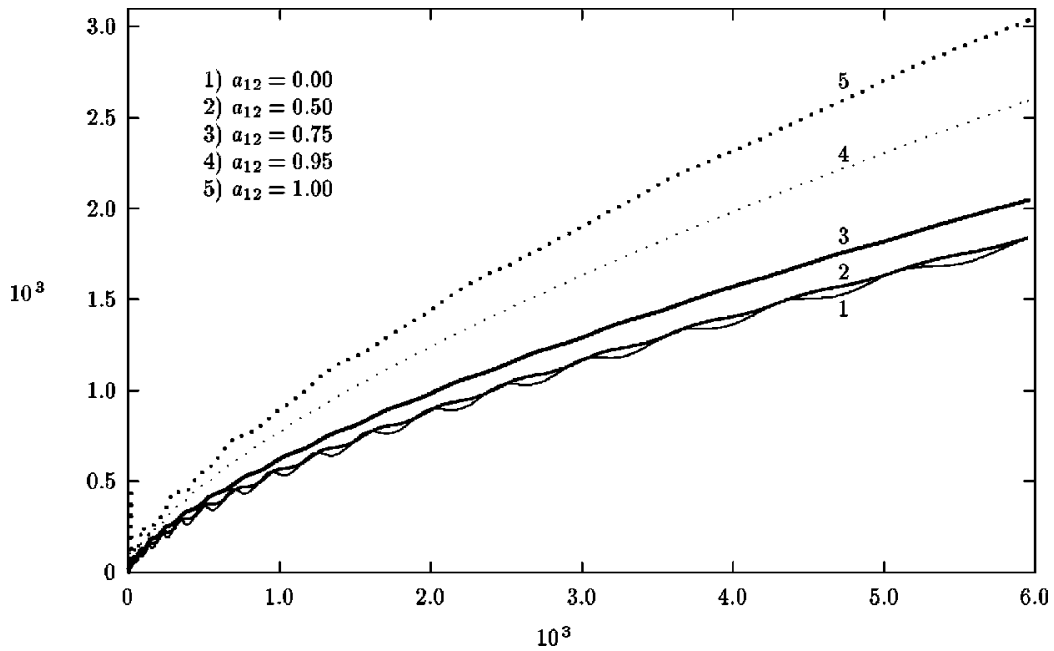

FIG. 7. $\widetilde{\Pi}(z, R / L)$, Eq. (65) as a function of particle density $z=2 N L^{2} / \pi$ for $R / L=0.5, a_{11}=a_{22}=1$, and five different values of $a_{12}$ $=0 ; 0.5 ; 0.75 ; 0.95 ; 1.0$.

$$
\begin{gathered}
\sigma=\frac{2 e^{2} L^{2}}{\pi^{4} l^{2}} \tilde{\Pi}\left(p_{F} R, R / L\right), \\
\widetilde{\Pi}=\frac{1}{45}\left(\frac{\pi}{2}\right)^{5 / 2} \frac{L^{4} R p_{F}^{5}}{a_{11}+a_{22}+2 a_{12}} .
\end{gathered}
$$

This curve for $R / L=50$ and $a_{12}=0$ is presented in Fig. 3 (thin line). The divergence between the quantum and quasiclassical curves is caused by a choice of the cutoff parameter and is deceptive. The cutoff parameter reconciles Eq. (90) with the main term $\nu^{5 / 2}$ in Eq. (80). As a result, the relative difference between the quasiclassical and quantum curves decreases with an increasing number of particles $z$, while the absolute value of this difference goes up as $\nu^{3 / 2}$.

For quasiclassical calculations in the intermediate range, one can use Eq. (53), which reproduces the limits (89) and (90), and provides a good approximation in-between. As it was mentioned earlier, the accuracy of the quasiclassical approach in this intermediate range is low.

\section{F. Effect of interwall correlations of inhomogeneities}

The effect of interwall correlations $a_{12} \neq 0$ on conductivity is determined by the oscillating term $(-1)^{j+j^{\prime}} a_{12}$ in transition probabilities (16) and (67) and strongly depends on $R / L$.

In general, the correlations of inhomogeneities affect the motion of particles either as a result of interference of consecutive "classical" collisions or as a result of a purely quantum multiple scattering on the scale of the particle wavelength. While the intrawall correlations are important only for the second effect, the interwall correlations can appear, depending on the parameters, as a result of both effects.

The consecutive scatterings from the opposite walls are correlated only to the extent to which the inhomogeneity $\xi\left(y_{2}, z_{2}\right)$ in the place of the second collision is correlated with the inhomogeneity $\xi\left(y_{1}, z_{1}\right)$ in the place of the first one. If the walls are very close, $R / L \gg 1$, the $(y, z)$ coordinates of both collisions are close to each other, and both scattering processes occur on the "same", inhomogeneity (if the inhomogeneities on the opposite walls are correlated). Then the transport coefficients in the second order in scattering should depend on the interwall correlations $a_{12}$ in the same way as on the intrawall ones. In agreement with this simple explanation, the conductivity at large values of $R / L$ is a monotonically decreasing function of $a_{12}$, Eq. (79) for all values of $S$, and the correlation amplitudes enter in the combination $a_{11}+a_{22}+2 a_{12}$. This result is illustrated in Fig. 3 (the solid line corresponds to $a_{11}=a_{22}=1, a_{12}=0$; the dotted line corresponds to $\left.a_{11}=a_{22}=1, a_{12}=0.75\right)$. Note, that in this case $\lambda<L \ll R$, and the "quantum" interference effects for interwall collisions are smaller than classical.

In the opposite case of $R / L \ll 1$, the $(y, z)$ coordinates of collisions with the opposite walls are separated from each other by a distance much larger than the size of the inhomogeneities. Therefore, the consecutive collisions are, on average, uncorrelated even if there is strong interwall correlations of inhomogeneities on the scale $R$. In this case, the dependence on the interwall correlations $a_{12}$ should disappear from the equations. The only exception is the ultraquantum case $\lambda \sim L$ when both consecutive collisions are within the same quantum "reaction zone" and, obviously, interfere with each other. Since the typical values of the wavelength $\lambda \sim 1 / p_{F}$ $\sim L / S$, the interwall correlations for $R / L \ll 1$ should be noticeable only for small $S$. This situation is illustrated by Eqs. (84) and (85) for $p_{F} R \ll 1$. At large $S$ (Fig. 4), the contribution from the interwall correlation amplitude $a_{12}$ has a purely oscillatory character and vanishes completely from the equations after the summation (averaging) over the energy levels $j^{\prime}$. When $S$ is small (Fig. 5), the summation over $j^{\prime}$ is insufficient to mask interwall correlations and the interwall contribution is an oscillating function of $S$ : the dotted line $\left(a_{12}=0.75\right)$ is either above or below the solid line $\left(a_{12}=0\right)$, depending on the value of $S$. At larger $S$ (Fig. 4), the dotted line becomes indistinguishable from the solid one.

Away from these limiting cases, the contribution from interwall correlations to the scattering probability is characterized by the factor $a_{12}(-1)^{j+j^{\prime}}$ without any general restrictions on relevant values of $j, j^{\prime}$. The resulting effect can be, depending on parameters, constructive, as in Eq. (79), destructive, or mixed, as in Fig. 5. The destructive interference is illustrated in Fig. 7 for five values of $a_{12}$ $=0 ; 0.5 ; 0.75 ; 0.95 ; 1.0$ and an intermediate value of $R / L$ 
$=0.5$. For this $R / L$, the singularities in the points when the number $S$ of the occupied bands changes are not well pronounced and are even more suppressed by interwall correlations. At $a_{12}=1$, the effect of interwall correlations can be anomalously large. The kernel of the transport equation, i.e., the transition probability $W$, contains the correlation amplitudes in combination $a_{11}+a_{22}+2 a_{12}(-1)^{j+j^{\prime}}$. If $a_{12}=1$, this kernel is zero for certain values of $j^{\prime}$ thus strongly affecting the solution. This is reflected in a rapid increase in conductivity when $a_{12} \rightarrow 1$.

\section{G. Comparison with other recent transport calculations}

Recent advances in nanotechnology, quantum wells, etc., brought into being numerous publications on transport in ultranarrow channels with rough boundaries. $^{19-23,25-32,34,40-42,50,51,52}$ Of these, only Refs. $23,25,28$, and 34 have quantum expressions for transport coefficients via the correlation function of surface roughness that can be compared to our previous ${ }^{40-42}$ and current results.

First, this work is the only one that reveals and explores the anomalous quantum regime for the transport equation. All previous publications use a standard transport equation. Second, we present a systematic analysis of the effect of interwall correlations on transport.

Away from the anomalous region and apart of interwall correlations, our result (84) for long wave particles $p_{F} R \ll 1$ at $a_{12}=0$ coincide with Eq. (13) of the first Ref. 23 and Eq. (3.13) of Ref. 28. Both papers do not contain many analytical or numerical results beyond this limit. Note that the case $p_{F} R \ll 1$ is not sensitive to the particular form of the correlation function and is, effectively, the case of $\delta$-type correlations (4) and quantum reflection.

Fishman and Calecki $^{23}$ replaced the quantum problem with random boundary conditions by a problem with random bulk Hamiltonian. The exact mapping is achieved by our Hamiltonian (7) with the matrix elements (10). The authors of Ref. 23 choose a much simpler Hamiltonian with the matrix elements that correspond to the first term in Eqs. (10) (with one rough wall). This turned out to be a good model: as we demonstrated in Sec. II, only this part of the exact matrix element contributes to the Boltzmann-Waldmann-Snider transport equations (13) and (17). Therefore, the transport equation of Ref. 23 was the same as our truncated equations (13) and (17), though with inhomogeneities on only one wall and, therefore, without interwall terms. Thus the authors of Ref. 23, might, in principle, obtain our analytical results outside the anomalous region and/or numerical results of Ref. 40. The quantum transport equation for the anomalous region or interwall terms cannot be reproduced by the method in Ref. 23 because of the simplified mapping of the boundary problem onto the bulk.

Kawabata $^{25}$ analyzed 1D channels with rough walls with the help of "adiabatic" wave functions $\Psi$ $\sim \exp (i q y) \sin \left\{\pi j\left[x+L / 2-\xi_{2}(y)\right] /\left[L-\xi_{1}(y)-\xi_{2}(y)\right]\right\} \quad$ (in the notations of our paper) without specifying the "adiabatic' conditions. The use of such a wave function requires a slow variation of shape along the channel, $q R \gg j, j l / L$. Though these conditions were sufficient for calculation of the matrix elements and the reflection coefficient, transport calculations in Ref. 25 required use of the perturbative
Boltzmann equation with an extra condition of the smallness of the wall corrugation. As a result, the transport restrictions in Ref. 25 are equivalent to supplementing our Eq. (8), $l$ $\ll R, L$, by a strong extra condition $q R \gg S$ which is similar to that of the limiting cases in our Secs. IV and V, $R \gg L$. This means that Ref. 25 completely misses the anomalous region and operates with the matrix elements (2.15) that coincide exactly with our Eq. (10) if Eq. (10) is simplified outside the anomalous region by using $\pi^{2} j^{2}+q^{2}=\pi^{2} j^{\prime 2}+q^{\prime 2}$. Transport calculations in Ref. 25 for the limiting case $R \gg L$ are performed only for one and two occupied minibands ( $S$ $=1 ; 2$ ) and are much less general than our analytical and numerical multiband results of Secs. IV and V for an arbitrary relation between $R$ and $L$. Reference 25 , in contrast to previous calculations, contains the interwall term in the matrix elements (2.15); however, the analysis of the effect of interwall correlations on transport is missing.

The authors of Ref. 28 used the $S$ matrix $^{37}$ for scattering by one random rough wall in order to derive the quasiclassical boundary condition for the distribution function. The authors solved the quasiclassical Boltzmann transport equation with this boundary condition, and calculated the conductivity (the low-momentum cutoff, in contrast to Sec. IV E, was fixed by impurity scattering). The transition to the quantum equations was achieved by replacing $p_{x}$ by $\pi j / L$ and summation over $j$ instead of integration. This procedure effectively replaced the inversion of matrices when solving the quantum matrix transport equation by quantization of the classically inverted operator. This approach is valid for $p_{F} R \ll 1$. In the presence of interband transitions, the results based on Ref. 28 should be much less accurate. In addition, the method in Ref. 28 cannot describe the effect of interwall correlations or the quantum transport equation for the anomalous region.

\section{SUMMARY AND DISCUSSION}

We performed a rigorous diagrammatic derivation of the transport equation for particles in thin films and narrow channels with random rough walls. Together with our technique of mapping of a transport problem with scattering by random surface inhomogeneities onto an equivalent bulk problem, these results present a consistent approach to (ballistic) transport between walls with slight random roughness.

The main finding is that the standard transport equation fails when the distance between quantized energy levels for the motion across the film $\Omega_{i j}$ is comparable to the wallinduced effective perturbation $\widehat{1 / \tau}$. In this anomalous quantum resonance region, the equations become more complicated than and differ from those for scattering by bulk impurities. This difference is explained by a peculiar operator form of the wall-induced effective perturbation. We analyzed the corresponding range of parameters and determined the magnitude of the anomaly by calculating the mobility of degenerate particles for the Gaussian correlation function of surface inhomogeneities.

In this anomalous range our previous coordinate transformation $^{40-42}$ should be modified to make the Jacobian $J=1$ and the perturbation operators Hermitian. This change brings unusual supplemental correlators of surface inhomogeneities into equations. Outside of this range of parameters, 
our original transformation leads to accurate results, and the supplemental correlators disappear.

Inside this special quantum range of parameters, the effective transport equation with wall scattering is extremely cumbersome. Beyond this range, the transport equation for particle scattering by random rough walls is essentially the same as for scattering by impurities and is relatively simple. According to Eqs. (73)-(75), the anomalous region corresponds to a moderately large number of occupied states $S$ for quantized finite motion across the film, when

$$
l^{2} S^{3 / 2} / L R \sim 1, \quad S>L^{2} / R^{2}, \quad l<R^{2} / L
$$

or

$$
l S^{3 / 2} R / L^{2} \sim 1, \quad 1<S<L^{2} / R^{2}, \quad R, L \gg l>R^{2} / L .
$$

This anomaly can be best observed for not very small amplitude of surface inhomogeneities $l$.

In addition to solving the transport equation with Gaussian correlations numerically in a wide range of parameters, we obtained analytical expressions for the transport coefficients in the limiting cases of long wave ballistic particles $p_{F} R \ll 1$ and very close walls $L / R \ll 1$ for both quantum and quasiclassical motion in the direction perpendicular to the walls and an arbitrary form of the correlation function.

The effect of interwall correlations of inhomogeneities on opposite walls is nontrivial. Depending on parameters, the interwall correlations can either decrease or increase particle mobility. Sometimes the effect of interwall correlations is an oscillating function of the number of occupied minibands $S$ depending on whether $S$ is odd or even. The effect of interwall correlations disappears for large $S$ except the case of the ultranarrow clearance between the walls.

The quasiclassical ballistic transport in thick films can retain certain quantum interference features related to a re- sidual constructive or destructive interference between the particles reflected from the opposite walls irrespective of how far apart are the walls. In this sense, the truly classical transport results for thick films should involve finite bulk attenuation.

The developed transport formalism provides a starting point for a study of the interference between wall and bulk scattering processes.

\section{ACKNOWLEDGMENTS}

This work was supported by NSF Grant Nos. DMR9412769 and DMR-9705304.

\section{APPENDIX A: TRANSITION PROBABILITY}

Equation (11) provides a simplified expression for the

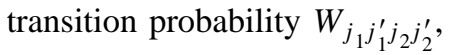

$$
\left\langle V_{\mathbf{q}_{1} j_{1}, \mathbf{q}_{1}^{\prime} j_{1}^{\prime}} V_{\mathbf{q}_{1}^{\prime} j_{2}, \mathbf{q}_{2}^{\prime} j_{2}^{\prime}}\right\rangle_{\xi}=(2 \pi)^{2} W_{j_{1} j_{1}^{\prime} j_{2} j_{2}^{\prime}}\left(\mathbf{q}_{1}, \mathbf{q}_{1}^{\prime}\right) \delta\left(\mathbf{q}_{1}-\mathbf{q}_{2}^{\prime}\right) .
$$

The full expression has the form $W=W^{(1)}+W^{(2)}$, where $W_{j_{1} j_{1}^{\prime} j_{2} j_{2}^{\prime}}^{(1)}\left(\mathbf{q}_{1}, \mathbf{q}_{1}^{\prime}\right)$ is given by Eq. (11) if the interwall correlation functions $\widetilde{\zeta}_{12}$ and $\widetilde{\zeta}_{21}$,

$$
\widetilde{\zeta}_{i k}(\mathbf{q})=\xi_{i}(\mathbf{q}) \widetilde{\xi}_{k}(-\mathbf{q}), \quad \widetilde{\xi}_{i}(\mathbf{q})=q \mathbf{e} \cdot \frac{\partial}{\partial \mathbf{q}} \int_{1}^{\infty} \xi_{i}(\alpha \mathbf{q}) \frac{d \alpha}{\alpha},
$$

exist and are real. If $\widetilde{\zeta}_{12}=\widetilde{\zeta}_{21}=0$, one should disregard the term $\widetilde{\zeta}_{11} \widetilde{\zeta}_{22} / \sqrt{\zeta_{11} \zeta_{22}}$ in Eq. (11). The second part of the transition probability, $W_{j_{1} j_{1}^{\prime} j_{2} j_{2}^{\prime}}^{(2)}\left(\mathbf{q}_{1}, \mathbf{q}_{1}^{\prime}\right)$, has the form

$$
\begin{aligned}
& W^{(2)}=\frac{\mathbf{q}_{1} \cdot\left[\mathbf{e} \times \mathbf{q}_{1}^{\prime}\right]}{2 m^{2} L^{2}} \frac{q_{1}^{\prime 2}-q_{1}^{2}}{\left(\mathbf{q}_{1}^{\prime}-\mathbf{q}_{1}\right)^{2}}\left[\widetilde{\zeta}_{11}(-1)^{j_{1}+j_{1}^{\prime}+j_{2}+j_{2}^{\prime}}+\widetilde{\zeta}_{22}+\widetilde{\zeta}_{12}+\widetilde{\zeta}_{21}(-1)^{j_{1}+j_{1}^{\prime}+j_{2}+j_{2}^{\prime}}\right] \\
& \times\left(\left\{\left[\left(\frac{\pi j_{1}}{L}\right)^{2}-\frac{1}{4} \frac{\left(q_{1}^{2}-q_{1}^{\prime 2}\right)^{2}}{\left(\mathbf{q}_{1}^{\prime}-\mathbf{q}_{1}\right)^{2}}\right] \delta_{j_{1} j_{1}^{\prime}}+\left(q_{1}^{2}-q_{1}^{\prime 2}\right) \frac{\left(1-\delta_{j_{1} j_{1}^{\prime}}\right) j_{1} j_{1}^{\prime}}{j_{1}^{\prime 2}-j_{1}^{2}}\right\} \delta_{j_{2} j_{2}^{\prime}}\right. \\
& \left.+\left\{\left[\left(\frac{\pi j_{2}}{L}\right)^{2}-\frac{1}{4} \frac{\left(q_{1}^{2}-q_{1}^{\prime 2}\right)^{2}}{\left(\mathbf{q}_{1}^{\prime}-\mathbf{q}_{1}\right)^{2}}\right] \delta_{j_{2} j_{2}^{\prime}}-\left(q_{1}^{2}-q_{1}^{\prime 2}\right) \frac{\left(1-\delta_{j_{2} j_{2}^{\prime}}\right) j_{2} j_{2}^{\prime}}{j_{2}^{\prime 2}-j_{2}^{2}}\right\} \delta_{j_{1} j_{1}^{\prime}}\right),
\end{aligned}
$$

where $\mathbf{e}$ is the unit vector perpendicular to the film, and the correlation functions $\widetilde{\zeta}_{i i}$ are defined by Eq. (12).

In principle, we can perform all the calculations with the full transition probability $W$, including the term (A2). Because of the factor $\mathbf{q}_{1} \cdot\left[\mathbf{e} \times \mathbf{q}_{1}^{\prime}\right]=q_{1} q_{1}^{\prime} \sin \chi$ in $W$, this term will result in the presence of the harmonics with sin $\theta$ in the expansion of the transport equation in the angular Fourier harmonics (42) and (44). Then we will have to look for the solution of the transport equation in the form

$$
\delta n_{j j^{\prime}}^{i}(\theta)=\frac{1}{2} \delta n_{j j^{\prime}}^{i(0)}+\sum_{s=1}^{\infty} \delta n_{j j^{\prime}}^{i(s)} \cos (s \theta)+\sin \theta \sum_{s=1}^{\infty} \delta \tilde{n}_{j j^{\prime}}^{i(s)} \cos (s \theta) .
$$

This will lead to a doubling of Eqs. (46), but will not result in any new physical effect. Since we do not know the correlation functions $\widetilde{\zeta}$, and assume, in computations, that $\widetilde{\zeta}=\zeta$ anyway, the use of Eq. (A2) will make the expressions for $W$ even more cumbersome than they already are without bringing in any new physical insight.

When the off-diagonal contributions are irrelevant and/or in the quasiclassical limit, the terms with $W^{(2)}$ [Eq. (A2)] automatically disappear from the equations because of the energy $\delta$ functions $\delta\left[\boldsymbol{\epsilon}_{j}(\mathbf{q})-\boldsymbol{\epsilon}_{j^{\prime}}\left(\mathbf{q}^{\prime}\right)\right]$ in the integrands. As a result, under these conditions the supplemental correlators $\widetilde{\zeta}$ become irrelevant. 
${ }^{1}$ P. Beckmann and A. Spizzichino, The Scattering of Electromagnetic Waves from Random Surfaces (Pergamon, New York, 1963).

${ }^{2}$ F. G. Bass and I. M. Fuks, Wave Scattering from Statistically Rough Surfaces (Pergamon, New York, 1979).

${ }^{3}$ J. A. DeSanto and D. S. Brown, Analytical Techniques for Multiple Scattering from Rough Surfaces, edited by E. Wolf (NorthHolland, Amsterdam, 1986).

${ }^{4}$ J. R. Willis, Mathematical Aspects of Scattering from Rough Cracks, in Mathematic Modelling in Non-Destructive Testing, edited by M. Blakemore and G. A. Georgiou (Clarendon, Oxford, 1988), p. 57.

${ }^{5}$ Interactions of Atoms and Molecules with Solid Surfaces, edited by V. Bortolani, N. H. March, and M. P. Tosi (Plenum, New York, 1990).

${ }^{6}$ J. A. Ogilvy, Theory of Wave Scattering from Random Surfaces (Adam Hilger, Bristol, 1991).

${ }^{7}$ A. G. Voronovich, Wave Scattering at Rough Surfaces (Springer, Berlin, 1994).

${ }^{8}$ A. R. McGurn, and A. A. Maradudin, Phys. Rev. B 30, 3136 (1984).

${ }^{9}$ P. Arseyev, Pis'ma Zh. Éksp. Teor. Fiz. 45, 132 (1987) [JETP Lett. 45, 163 (1987)].

${ }^{10}$ L. I. Glazman, G. B. Lesovik, D. E. Khmel'nitskii, and R. I. Shekhter, Pis'ma Zh. Éksp. Teor. Fiz. 48, 218 (1988) [JETP Lett. 48, 238 (1988)].

${ }^{11}$ D. A. Wharam et al., J. Phys. C 21, L209 (1988).

${ }^{12}$ B. J. van Wees et al., Phys. Rev. Lett. 60, 848 (1988).

${ }^{13}$ V. I. Kozub and A. A. Krokhin, J. Phys.: Condens. Matter 5, 9135 (1993).

${ }^{14}$ P. A. Lee and T. V. Ramakrishnan, Rev. Mod. Phys. 57, 287 (1985).

${ }^{15}$ B. Altshuler, in Nanostructures and Mesoscopic Systems, edited by W. P. Kirk and M. A. Reed (Academic Press, New York, 1991), pp. 405-416.

${ }^{16}$ C. Itzykson and J.-M. Drouffe, Statistical Field Theory (Cambridge University Press, Cambridge, 1991), Vol. 2, Chap. 10.

${ }^{17}$ V. I. Okulov and V. V. Ustinov, Sov. J. Low Temp. Phys. 5, 101 (1979).

${ }^{18}$ T. Ando, A. B. Fowler, and F. Stern, Rev. Mod. Phys. 54, 437 (1982).

${ }^{19}$ R. Lenk and A. Knabchen, J. Phys.: Condens. Matter 5, 6563 (1993).

${ }^{20}$ P. M. Levy, S. Zhang, and A. Fert, Phys. Rev. Lett. 65, 1643 (1990).

${ }^{21}$ A. C. Ehrlich, Phys. Rev. Lett. 71, 2300 (1993).

${ }^{22}$ G. Reiss, E. Hastreiter, H. Bruckl, and J. Vancea, Phys. Rev. B 43, 5176 (1991)

${ }^{23}$ G. Fishman and D. Calecki, Phys. Rev. Lett. 62, 1302 (1989); Phys. Rev. B 43, 11581 (1991).

${ }^{24}$ Y. Takagaki and D. K. Ferry, J. Phys.: Condens. Matter 4, 10421 (1992).

${ }^{25}$ A. Kawabata, J. Phys. Soc. Jpn. 62, 3988 (1993).

${ }^{26}$ K. Nicolic and A. McKinnon, Phys. Rev. B 50, 11008 (1994).

${ }^{27}$ A. A. Krokhin, N. M. Makarov, and V. A. Yampl'skii, J. Phys.: Condens. Matter 3, 4621 (1991).
${ }^{28}$ N. M. Makarov, A. V. Moroz, and V. A. Yampol'skii, Phys. Rev. B 52, 6087 (1995).

${ }^{29}$ B. G. Orr, H. M. Jaeger, and A. M. Goldman, Phys. Rev. B 32, 7586 (1985)

${ }^{30}$ C. W. J. Beenakker and H. van Houten, Phys. Rev. Lett. 63, 1857 (1989).

${ }^{31}$ K. Nakazato and R. J. Blaikie, J. Phys.: Condens. Matter 3, 5729 (1991).

${ }^{32}$ E. Z. Luo, S. Heun, M. Kennedy, J. Wollschlager, and M. Henzler, Phys. Rev. B 49, 4858 (1994).

${ }^{33}$ Z. L. Wang, H. Ogura, and N. Takahashi, J. Opt. Soc. Am. A 12, 1489 (1995).

${ }^{34}$ G. Palasantzas and J. Barnas, Phys. Rev. B 56, 7726 (1997).

${ }^{35}$ A. V. Chaplik and M. V. Entin, Zh. Éksp. Teor. Fiz. 55, 990 (1968) [Sov. Phys. JETP 28, 514 (1969)].

${ }^{36}$ L. A. Falkovskii, Zh. Éksp. Teor. Fiz. 58, 1830 (1970) [Sov. Phys. JETP 31, 981 (1970)]; 60, 838 (1971) [33, 454 (1971)].

${ }^{37}$ A. G. Voronovich, Sov. Phys. Acoust. 30, 444 (1984); Zh. Éksp. Teor. Fiz. 89, 116 (1985) [Sov. Phys. JETP 62, 65 (1985)].

${ }^{38}$ E. I. Thorsos and D. R. Jackson, J. Acoust. Soc. Am. 86, 261 (1989).

${ }^{39}$ F. Bass, V. D. Freulicher, and I. Fuks, IEEE Trans. Antennas Propag. 22, 278 (1974); 22, 288 (1974).

${ }^{40}$ A. E. Meyerovich and S. Stepaniants, J. Phys.: Condens. Matter 9, 4157 (1997).

${ }^{41}$ A. E. Meyerovich and S. Stepaniants, Phys. Rev. Lett. 73, 316 (1994).

${ }^{42}$ A. E. Meyerovich and S. Stepaniants, Phys. Rev. B 51, 17116 (1995).

${ }^{43}$ J. A. Konrady, Jr., J. Acoust. Soc. Am. 56, 1687 (1974).

${ }^{44}$ H. D. I. Abrabanel, J. Acoust. Soc. Am. 68, 1459 (1980).

${ }^{45}$ J. G. Watson and J. B. Keller, J. Acoust. Soc. Am. 75, 1705 (1984).

${ }^{46}$ E. I. Thorsos and D. R. Jackson, J. Acoust. Soc. Am. 86, 261 (1989).

${ }^{47}$ R. E. Goldstein, A. I. Pesci, and V. Romero-Rochin, Phys. Rev. A 41, 5504 (1990).

${ }^{48}$ A. B. Isers, A. A. Puzenko, and I. M. Fuks, J. Electromagn. Waves Appl. 5, 1419 (1991).

${ }^{49}$ D. H. Berman, J. Acoust. Soc. Am. 92, 309 (1992); 96, 417 (1994).

${ }^{50}$ Z. Tesanovic, M. V. Jaric, and S. Maekawa, Phys. Rev. Lett. 57, 2760 (1986).

${ }^{51}$ N. Trivedi and N. W. Ashcroft, Phys. Rev. B 38, 12298 (1988).

${ }^{52}$ A. M. Bratkovsky and S. N. Rashkeev, Phys. Rev. B 53, 13074 (1996).

${ }^{53}$ Z. Tesanovic, J. Phys.: Condens. Matter 20, L829 (1987).

${ }^{54}$ H. Moraal, Phys. Rep. 17, 225 (1975).

${ }^{55}$ E. M. Lifshitz and L. P. Pitaevski, Physical Kinetics (Plenum, New York, 1979).

${ }^{56}$ A. A. Abrikosov, L. P. Gor'kov, and I. E. Dzyaloshinsli, Methods of Quantum Field Theory in Statistical Physics (Dover, New York, 1975), Sec. 39.2.

${ }^{57}$ S. F. Edwards, Philos. Mag. 3, 1020 (1958).

${ }^{58}$ A. E. Meyerovich and K. A. Musaelian, J. Low Temp. Phys. 89, 781 (1992); Phys. Rev. Lett. 72, 1710 (1994). 